Baratas, Luis. Distribución funcional de la renta: análisis de la divergencia en la participación salarial entre Alemania y España 1999 - 2013.

\title{
DISTRIBUCIÓN FUNCIONAL DE LA RENTA: ANÁLISIS DE LA DIVERGENCIA EN LA PARTICIPACIÓN SALARIAL ENTRE ALEMANIA Y ESPAÑA 1999 - 2013
}

\section{FUNCTIONAL DISTRIBUTION OF INCOME: ANALYSIS OF THE DIVERGENCE ON THE WAGE SHARE BETWEEN GERMANY AND SPAIN 1999 - 2013}

\author{
LUIS BARATAS \\ Universidad Complutense de Madrid \\ luisbaratasgonzalez@ucm.es
}

Fecha de recepción: octubre de 2015 Fecha de aceptación: noviembre de 2015

\begin{abstract}
RESUMEN
Este trabajo plantea un análisis empírico de la evolución experimentada por la distribución funcional de la renta en las economías de España y Alemania entre 1999 y 2013. El hecho destacado sobre el que pretendemos arrojar luz es la divergencia en la evolución de la participación salarial que da comienzo entre ambos países a partir del año 2010. Para ello el trabajo ofrece previamente un repaso bibliográfico del que se extraen las principales variables que autores de la corriente de Economía Política han identificado como plausibles determinantes de la participación salarial. Una vez identificados los factores que determinan la evolución de nuestra variable dependiente se procede a contextualizarlos en el contexto de la Unión Económica y Monetaria Europea en el que se encuentran España y Alemania a lo largo del periodo. Ante las variables explicativas seleccionadas y los resultados empíricos obtenidos la conclusión principal que sostiene este trabajo es que las razones de la divergencia en la participación salarial se sitúan en el débil crecimiento de la productividad y la fuerte reducción del salario medio en España. A su vez, aprovechamos para apuntar varias posibles explicaciones a la divergencia en las participaciones salariales (débil estructura productiva y los cambios en la negociación colectiva en parte como consecuencia de la puesta en marcha del euro) ${ }^{1}$.
\end{abstract}

Palabras clave: participación salarial, productividad, salarios, coste laboral unitario, estructura productiva, negociación colectiva.

\section{ABSTRACT}

This paper presents an empirical approach to the evolution undergone by the functional distribution of income in the economies of Spain and Germany between 1999 and 2013. The striking fact about which we intend to shed light is the

\footnotetext{
${ }^{1}$ Una versión preliminar de este trabajo fue presentada el 25 de junio de 2015 en el 12th International Conference Developments in Economic Theory and Policy organizado por la Universidad del País Vasco y la Universidad de Cambridge en Bilbao los días 25 y 26 de junio de 2015.
} 
Baratas, Luis. Distribución funcional de la renta: análisis de la divergencia en la participación salarial entre Alemania y España 1999 - 2013.

divergence in the evolution of the wage share that starts between the two countries since 2010 . To do this, the work previously provides a literature review of the main contributions of authors from Political Economics approach have been identified as plausible determinants of the wage share. Once we have identified factors that determine the evolution of our dependent variable we proceed to contextualize them in the scenario where Spain and Germany are over period. Given the explanatory variables chosen and the empirical results, the main assumptions that sustains this work is that the reasons for the divergence in wage share are located in the weak productivity gains and the strong reduction of the average wage in Spain. In turn, we took the opportunity to aim some possible explanations for the divergence in wage shares (weak production structure and changes in collective bargaining, in part due to the launch of the euro).

Key words: wage share, productivity, wages, unit labor costs, productive structure, and collective bargaining.

JEL: E20, E24, E25, E30, E50

\section{INTRODUCCIÓN}

Es un hecho constatable que la distribución funcional de la renta (DFR) de una economía puede experimentar variaciones en su patrón distributivo entre salarios y beneficios a lo largo del tiempo. En concreto, la participación de los salarios sobre la renta en las economías desarrolladas viene experimentando una progresiva caída desde los años ochenta hasta nuestros días, después de un periodo de gradual aumento durante las décadas de los cincuenta, sesenta y setenta.

Desde una parte de la literatura académica sencillamente se ha obviado este fenómeno por la casi nula atención prestada a la DFR como objeto de estudio asumiendo la hipótesis de que la misma se explica en base a la productividad marginal de cada factor productivo. Mientras tanto otra parte de la literatura económica que arranca en autores clásicos como Ricardo, sí han concedido gran importancia al análisis de la DFR tanto desde el punto de vista de sus determinantes como del de sus implicaciones para la dinámica de acumulación. De entre estas dos cuestiones, nuestro trabajo se enmarca en la primera de ellas, es decir, en el debate sobre las causas que determinan la evolución de la DFR, por ser la menos estudiada de las dos. Como podremos ver en el apartado dos, existen diferentes respuestas a esta cuestión y, por ello, el primer objetivo de este trabajo es presentar, de forma muy sintética, las principales aportaciones que se han hecho en este sentido, proponiendo una clasificación que nos ayude a identificar las principales semejanzas y diferencias entre ellas así como extraer algún tipo de conclusión que, a modo de hipótesis, guíe nuestro posterior estudio de caso.

El segundo objetivo de nuestro trabajo, y a partir del cual se construye la que consideramos la principal aportación de este TFM, es el análisis comparado de la DFR para las economías de España y Alemania durante el periodo 1999-2013 Aun sufriendo una similar y paulatina pérdida de cuota salarial en las últimas décadas, el desarrollo económico de estas economías desde la entrada en el 
Baratas, Luis. Distribución funcional de la renta: análisis de la divergencia en la participación salarial entre Alemania y España 1999 - 2013.

euro ha sido bastante diferente. Este desarrollo ha venido caracterizado por las consecuencias derivadas de la pérdida de control de la herramienta del tipo de cambio, la aplicación de reformas estructurales especialmente en el mercado de trabajo y la adopción de estrategias competitivas de carácter rival. El paso de la crisis económica por Europa recalcó la fragilidad de la construcción monetaria europea que -de entre muchas cosas- se ha escenificado en un comportamiento divergente de la participación salarial (PS).

Gráfico 1. Participación salarial: UE28 y países, 1999 - 2013

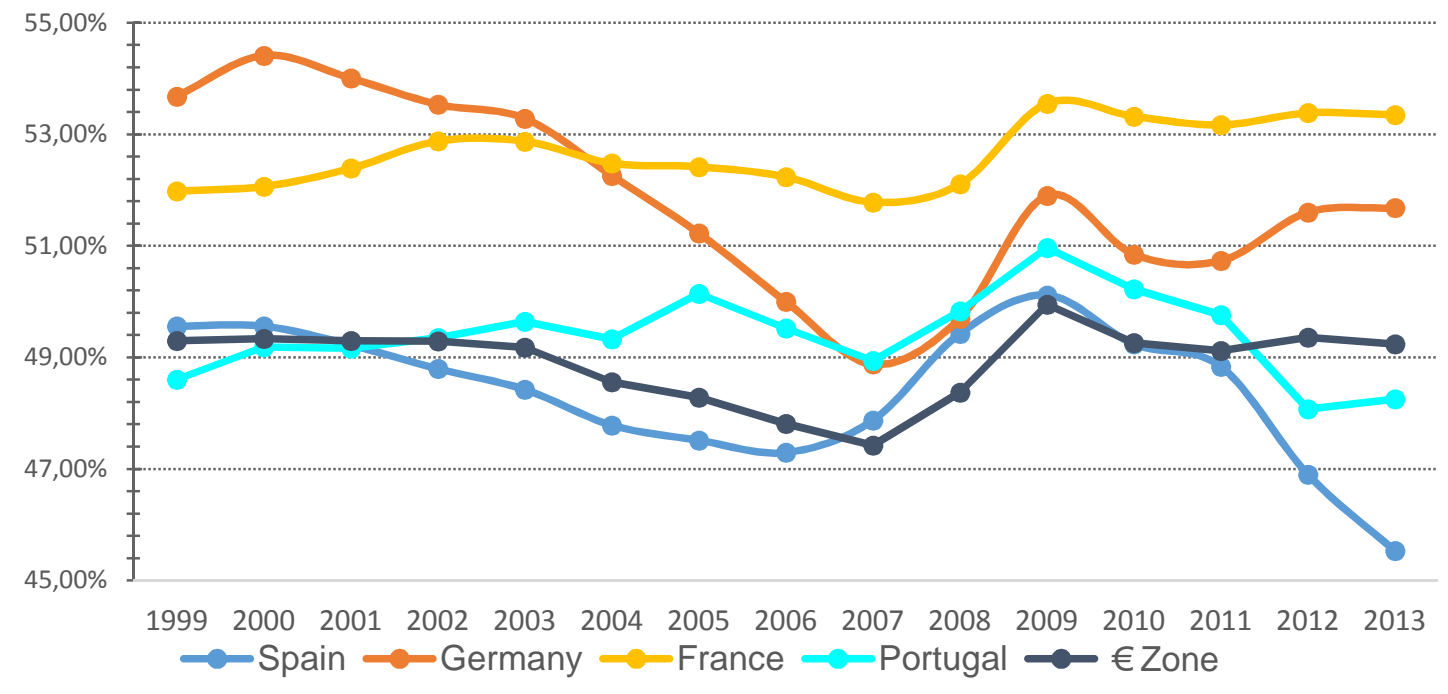

Fuente: elaboración propia a partir de datos de AMECO.

Mientras que en Alemania desde 2010 se detiene la pérdida de cuota de los salarios, incluso crece tímidamente, en España esa pérdida de participación es cada vez mayor. Ante un escenario así nos preguntamos sobre las raíces de esos comportamientos dispares. Lo que podríamos asumir como previsible para dos economías desarrolladas que forman parte de una misma unión económicamonetaria, y que históricamente han experimentado una evolución similar, sería que la PS se comportase de manera relativamente similar en ambos países. Aunque, por otro lado, la existencia de desequilibrios estructurales dentro de la propia Unión y la necesidad de aplicar devaluaciones salariales por la imposibilidad de que sean monetarias, sienta las bases para que no podamos asumir con toda certeza esa previsión. Ante esta aparente contradicción argumental y la evidente discordancia en las participaciones salariales después de la crisis cabe preguntarse si la evolución en un país y otro responde a los mismos factores. Por tanto el segundo, y más importante, de los objetivos de este trabajo será analizar las tendencias generales de la PS y de sus componentes, y localizar las raíces de esa divergencia en el comportamiento de la DFR a partir de 2010.

Tras el estallido de la crisis financiera que arrastró a la mayoría de las economías europeas a una profunda recesión, buena parte de las medidas adoptadas para 
Baratas, Luis. Distribución funcional de la renta: análisis de la divergencia en la participación salarial entre Alemania y España 1999 - 2013.

salir de la misma fueron encaminadas a ganar competitividad mediante la devaluación salarial. No es descabellado suponer que esta estrategia competitiva, junto con la debilidad endémica de la estructura productiva española, han tenido una incidencia directa sobre la evolución de la PS. De manera que, a medio camino entre la consecución del objetivo uno y el objetivo dos señalados anteriormente, el trabajo ofrece una contextualización de los hechos vividos en España y Alemania encaminada a sostener, tanto de manera explícita como implícita a lo largo de todo el trabajo, una primera explicación del comportamiento dispar de la PS entre España y Alemania en base a las modificaciones institucionales que han tenido lugar en el mercado de trabajo, particularmente en materia de negociación colectiva, junto con la crónicamente débil estructura productiva de la economía española.

Con la intención de acercarnos a la consecución de dichos objetivos, el trabajo está estructurado de la siguiente manera: un apartado de cuestiones teóricas y metodológicas, donde se comienza con una revisión de literatura priorizando el enfoque de economía política sobre el hegemónico enfoque neoclásico a la hora de analizar la DFR. Posteriormente se explican las cuestiones metodológicas asociadas al indicador de la DFR y a las magnitudes utilizadas en el análisis empírico. El tercer punto del trabajo corresponde a una contextualización estructural de nuestro objeto de estudio donde se resaltarán los factores particulares de estas economías en función de lo establecido en la revisión de literatura. El apartado cuarto concierne propiamente al análisis empírico de la PS y sus principales componentes. En este apartado se plantea la consecución del objetivo principal del trabajo, arrojando luz acerca del comportamiento de la productividad, el salario medio, el coste laboral unitario y la tasa de asalarización de las economías española y alemana. Por ultimo finalizaremos con un quinto apartado de conclusiones. Aunque en el tercer apartado de contextualización se apuntan diversas posibles explicaciones para la divergencia de las participaciones salariales y, en concreto, para las tendencias identificadas en el apartado cuatro, queda fuera de los límites de esta investigación llevar a cabo una contrastación en sentido estricto de dichas explicaciones.

\section{CUESTIONES TEÓRICAS Y METODOLÓGICAS}

\subsection{Revisión de literatura}

Es importante que recalquemos nuestro interés por estudiar la problemática de la distribución desde una óptica macroeconómica, la cual nos ayuda a comprender la dinámica general del sistema económico bajo el que vivimos. En contraposición a la óptica microeconómica que nos ofrece una visión más reducida e individualizada de las relaciones que tienen lugar en el mismo. Es por ello por lo que hemos decidido centrar nuestro trabajo sobre la distribución de la renta en su dimensión factorial, aquella mediante la cual se pueden comprender los procesos de acumulación, los flujos de ahorro-inversión, de oferta-demanda de empleo, en definitiva, del crecimiento de una economía tanto en el corto como en el largo plazo (Laborda 2001). Dejamos, por tanto, fuera de nuestro trabajo la dimensión personal de la distribución de la renta que arroja luz acerca de cuán 
Baratas, Luis. Distribución funcional de la renta: análisis de la divergencia en la participación salarial entre Alemania y España 1999 - 2013.

equitativa es una sociedad. Aun considerándola una dimensión muy importante y socialmente deseable, no encaja con las ambiciones de este trabajo.

En este repaso bibliográfico recopilaremos las contribuciones más destacadas que, tanto autores clásicos como contemporáneos, han aportado a la literatura sobre distribución funcional de la renta. Con él tenemos la intención de dar respuesta a la pregunta que numerosos economistas se vienen haciendo recientemente: ¿Qué factores explican las variaciones experimentadas por la participación de los salarios sobre el PIB en las últimas décadas? La estructura que seguirá este apartado estará marcada por la división entre la literatura teórica y empírica. En primer lugar prestaremos atención a la literatura teórica de autores del siglo XIX y XX. Esta literatura se puede dividir, a su vez, en dos grandes enfoques en tanto que atribuyen o no una connotación social y de clase a su análisis de la distribución. Estos enfoques son el de economía política y el enfoque neoclásico, nosotros le daremos una importancia central al primero de ellos al considerar que sus elementos teóricos se ajustan mucho mejor a la realidad y, por tanto, goza de una mayor capacidad explicativa para abordar una problemática como la que planteamos que el enfoque neoclásico. En segundo lugar atenderemos a la literatura empírica de las últimas décadas del siglo XX y primeras del XXI. A su vez en esta literatura podremos identificar tres corrientes diferentes: autores que no mantienen dicha connotación social, autores que sí mantienen dicha connotación y relacionan directamente sus investigaciones con algunas de las teorías anteriormente citadas y autores que aun manteniendo la connotación social y de clase no se apoyan directamente en los trabajos teóricos. El apartado servirá para ubicar teóricamente este trabajo en el debate sobre los determinantes de la DFR y para identificar los factores explicativos que cada uno de los autores considera pertinentes para dar respuesta a la pregunta inicial.

La cuestión de las leyes que configuran un determinado reparto del producto social entre factores productivos ha sido históricamente, desde el siglo XIX, el núcleo central de las preocupaciones de los economistas clásicos. La mayoría de ellos abordaban esta temática bajo una noción de estratificación de la sociedad en clases sociales y asumían que, en primera instancia -antes de pasar a aspectos técnicos-, el resultado final de la distribución factorial estará condicionado por la correlación de fuerzas existente entre clases. A los economistas, tanto clásicos como contemporáneos, que suscriben esta noción los incluimos bajo el paraguas de un mismo enfoque analítico que utilizaremos como referencia para nuestros argumentos, el de la economía política.

Este primer bloque de repaso bibliográfico lo iniciamos con David Ricardo (17721823). En su obra On the Principles of Political Economy and Taxation (1817) afirma que: "el principal problema de la economía política es el de determinar las leyes que configuran la distribución de la riqueza generada por una sociedad". Ricardo no se encarga de identificar factores explicativos de la variación de la PS, sino de vertebrar, en base a dos conceptos, su propia interpretación de las leyes que rigen la distribución de la renta. Por un lado, el de los rendimientos a escala decrecientes de la tierra, por el que los incrementos del producto agrario eran cada vez menores conforme se ampliaba la extensión cultivada (ante la 
Baratas, Luis. Distribución funcional de la renta: análisis de la divergencia en la participación salarial entre Alemania y España 1999 - 2013.

creciente peor calidad de las nuevas tierras). Dedujo que la renta que obtendrían los terratenientes sería, por tanto, el resultado de la diferencia entre la capacidad productiva de sus propias tierras -explotadas por trabajadores y/o capitalistas- y la capacidad productiva de las tierras marginales (libres). El segundo concepto básico se basaba en la idea de renta de subsistencia, que serviría para denotar el salario que obtendrían los trabajadores como aquella cantidad mínima que cubriría sus necesidades vitales. La situación que describe Ricardo se corresponde con lo que él mismo llama "estado estacionario" en el que la relación capital-trabajo permanece constante a lo largo del tiempo y los capitalistas/terratenientes no tienen incentivos para dinamizar la economía mediante procesos de inversión. Es preciso recordar que el periodo histórico a lo largo del cual David Ricardo elabora su obra es el de la Inglaterra de finales del siglo XVIII y primeras décadas del siglo XIX, momento en el que la sociedad agraria entra en regresión y se produce el tránsito hacia una economía industrial.

Por otro lado aludimos a la figura prominente de la corriente marxista, Karl Marx (1818-1883), del cual debemos rescatar su jerarquización de la sociedad en dos clases sociales, trabajadores y capitalistas. Introducirá, también, el concepto de plusvalía, entendido como la proporción del valor creado por parte del trabajador que se apropia el empresario. En su ampliamente extendida hipótesis sobre el "descenso tendencial de la tasa de ganancia", el aumento de la plusvalía actúa como factor contrarrestante de dicho declive. Por tanto el incremento de la tasa de plusvalía ${ }^{2}$, en aras de una ampliación de los beneficios, se convierte en un elemento susceptible de modificar la forma final que adoptará la DFR, en concreto, en un factor desencadenante de una progresiva pérdida de participación relativa de los salarios.

Conforme avanzó la historia del pensamiento económico se fue diluyendo el interés por la distribución funcional de la renta y su análisis desde una perspectiva de clase. La respuesta a este escaso acervo documental, en la línea que nos tenía acostumbrados la economía política, la encontramos en la aceptación generalizada, en buena parte del mundo académico, de la teoría marginalista ${ }^{3}$. Estos economistas encuadrados en la escuela neoclásica pasaron a interpretar las leyes de la distribución del ingreso dejando de lado cualquier componente de clase y centrando su explicación únicamente en los diferenciales de productividad entre los factores de producción capital y trabajo. Además lo hicieron bajo los irreales supuestos de competencia perfecta y plena utilización de los factores productivos que subyacían en la hegemónica función de producción Cobb-Douglas.

Las críticas a esta función de producción están sistematizadas por Felipe y McCombie $(2005,2014)$ en dos sentidos. Por un lado reprochan la falta de

\footnotetext{
${ }^{2}$ La tasa de plusvalía se puede incrementar: 1) de manera extensiva, cuando se incrementa la jornada laboral de los trabajadores (plusvalía absoluta) o 2) de manera intensiva, cuando el empresario fuerza un aumento de la intensidad del trabajo que reporta una generación superior de valor en el mismo tiempo o el mismo nivel en menor tiempo (plusvalía relativa). En ambos casos se incrementa la tasa de plusvalía.

${ }^{3}$ El origen de este enfoque dominante se asienta en los planteamientos de los primeros teóricos marginalistas que estudiaron cuestiones referentes a la distribución del ingreso como Stanley Jevons (1871) y John Bates Clark (1899).
} 
Baratas, Luis. Distribución funcional de la renta: análisis de la divergencia en la participación salarial entre Alemania y España 1999 - 2013.

exactitud en la medición del nivel de producción. Ya que para hacerlo con exactitud se deberían utilizar datos que aludiesen a las cantidades físicas tanto de inputs como de outputs, y no mediciones monetarias con el fin de poder homogeneizar mercancías heterogéneas. Por otro lado manifiestan la incoherencia entre la elasticidad de los factores productivos con respecto al producto y la participación relativa de cada uno de estos factores. Según la función de producción neoclásica las elasticidades del trabajo y capital son matemáticamente equiparables a las participaciones relativas de cada uno de estos factores sobre el PIB. Esta construcción augura unas elasticidades constantes del trabajo y el capital, por medio del establecimiento del precio a través de un margen por encima de los costes laborales, que conducirían a una participación relativa de cada factor productivo constante en el tiempo. Esta implicación entra fuertemente en contradicción con la posibilidad de variación de la DFR, y en especial con el hecho estilizado que venimos observando en las últimas décadas -y detallaremos en el siguiente apartado- de fuerte descenso de la participación relativa del factor trabajo.

Tras las bases analíticas que sientan Ricardo y Marx y la manifiesta infertilidad de las tesis neoclásicas se persiste en el interés de estudiar la distribución funcional de la renta desde un punto de vista de economía política. No por ello sin discrepancias entre autores. Estas discrepancias dieron lugar a varias líneas argumentales que, según nos interesa para acercarnos a la problemática bajo la concepción social de la que partimos, podemos sintetizar en marxista y keynesiana/poskeynesiana. Muy a groso modo, la primera de ellas asentaba sus análisis en el hecho fehaciente de las relaciones entre clases, mientras que la segunda, sin negar la existencia de las mismas, lo hacía en base a la dinámica de crecimiento de las economías.

Partiendo del estudio de los componentes de la demanda agregada John Maynard Keynes (1883-1946) sentó las bases de una renovada corriente analítica desde la que afrontar la problemática de la distribución. Primero en su obra Treatise on Money (1930), y posteriormente en The General Theory of Employment, Interest and Money (1936), conforma no una teoría definitiva de la distribución, pero sí -al menos- unas directrices básicas que servirían como punto de partida para autores ulteriores ${ }^{4}$. Siguiendo a Keynes, el beneficio del conjunto de la economía dependería de cuatro tipos de decisiones: i) Decisiones de inversión por parte de la clase empresarial, ii) Decisiones de consumo por parte de la clase trabajadora con mayor incidencia de la que pueda ejercer la clase empresarial-, iii) Decisiones de liquidez por parte de la sociedad en su conjunto y iv) Decisiones monetarias por parte de la autoridad de política monetaria. En mayor o menor medida, desde este punto partirán los sucesivos autores en sus teorías de la distribución.

En un intento de aplicar las leyes de la Teoría General de Keynes, el científico social -más que economista5- Kenneth Ewart Boulding (1910-1993) elaboró en 1950 un modelo de distribución partiendo de las identidades básicas de la

\footnotetext{
${ }^{4}$ Aunque algunos autores utilicen a Keynes como punto de partida para sus respectivos análisis, existen otros, como Sidney Weintraub (1914-1983), que se apoyan en él para llevar a cabo una síntesis distributiva que ponga en línea los planteamientos de Keynes con los de la escuela neoclásica. Weintraub $(1958,1959)$. ${ }^{5}$ Vida y obra recogida en Carpintero, O. (2012). Kenneth E. Boulding, más allá de la economía.
} 
Baratas, Luis. Distribución funcional de la renta: análisis de la divergencia en la participación salarial entre Alemania y España 1999 - 2013.

contabilidad nacional. A través de él logró extraer algebraicamente las ecuaciones que determinarían el peso de los salarios y los beneficios sobre la renta nacional. Siguiendo al propio Boulding, de su modelo se puede inferir que la DFR depende: en primer lugar de la composición de la absorción del producto, es decir de que parte se destina a inversión y que parte a consumo; y en segundo lugar de un factor de transferencia de rentas entre las unidades de producción y de consumo, en definitiva entre las clases sociales. De manera que el descenso de la PS puede venir provocado por una reducción de las transferencias a las unidades de consumo y/o una creciente dedicación del excedente neto a procesos inversores intensivos en capital. A pesar de ello, como bien indica el propio Boulding en su obra "este modelo solo será válido si los parámetros se adaptan de forma regular a los cambios en el comportamiento humano" (Boulding, 1950).

Una de las figuras más relevantes que investigó sobre la distribución del ingreso en una sociedad fue Michal Kalecki (1899-1970). Este economista polaco coetáneo a Keynes realizó algunas de las aportaciones más transcendentales a la teoría económica de la distribución cuando vinculó el grado de monopolio de una economía con la forma final que adoptaría la DFR. Con la intención de explicar esas lógicas de distribución internas entre salarios y beneficios, Kalecki parte de un análisis microeconómico de las empresas como unidades económicas, para posteriormente extrapolarlo a la economía en su conjunto. Según Kalecki $(1938,1954)$ las empresas que operan en el sistema capitalista lo hacen bajo una infrautilización de la capacidad productiva del capital instalado y con un cierto grado de monopolio (asumiendo la explicación de A. Lerner, 1934), por lo que el tamaño de los beneficios vendrá determinado por el ratio capital-trabajo y por un 'mark-up' que permite a los empresarios, a través de un determinado poder de mercado, establecer el precio de venta de sus productos. Por tanto la competencia existente en el sistema es de carácter imperfecto, algo que afecta ineluctablemente a la distribución del producto final. Mientras tanto, por el lado de los salarios Kalecki vaticina que la participación de los salarios en la renta vendrá determinada por una función inversa del grado de monopolio, es decir:

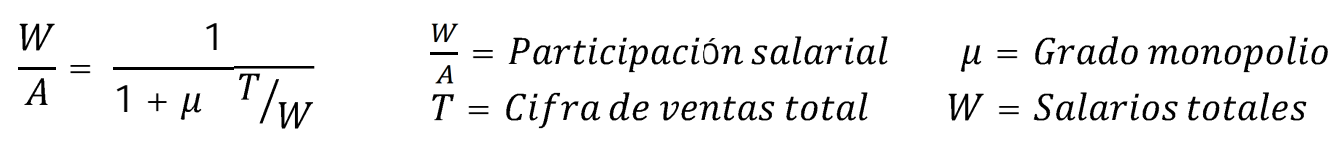

Las investigaciones de Kalecki suponen una contribución importantísima a la teoría de la distribución al interpretar el grado de monopolio, dentro de una dinámica de crecimiento económico ${ }^{6}$, como un factor explicativo de la progresiva pérdida de participación relativa de los salarios sobre el PIB. Si bien estos planteamientos han sufrido críticas por parte de otros autores como N. Kaldor, los cuales se tachan de tautológicos, o al menos, demasiado vagos para ser útiles (King, 2009: 72).

\footnotetext{
${ }^{6}$ Un estudio más íntegro sobre la relación dinámica de crecimiento-distribución funcional lo podemos encontrar en el famoso modelo de crecimiento que realizaron Badhuri y Marglin en 1990.
} 
Baratas, Luis. Distribución funcional de la renta: análisis de la divergencia en la participación salarial entre Alemania y España 1999 - 2013.

Este economista húngaro nacionalizado británico, Nicolás Kaldor (1908-1986), fue uno de los máximos exponentes de la renovada corriente poskeynesiana en materia de distribución. Su famoso artículo Alternative Theories of Distribution (1956) se trata de uno de los modelos teóricos y analíticos más acabados al respecto. En este artículo Kaldor se desmarca de los planteamientos previos elaborados por Kalecki y pasa a teorizar sobre el papel que juega la dinámica de distribución del ingreso de una economía en su proceso de crecimiento (Verduzco, 1998 pp 144).

Para ello parte de la igualdad entre ahorro e inversión de una economía que se caracteriza por el pleno empleo, donde la renta nacional es de carácter exógeno configurada por unos determinados niveles de stock de capital y de productividad técnica. En esta situación un incremento de la inversión para dinamizar el crecimiento económico supone, necesariamente, un trasvase de renta desde el grupo con una menor propensión al ahorro (trabajadores) hacia el de una mayor propensión al mismo (empresarios). De modo que, ante la permanencia fija -en el máximo posible- de las cantidades de trabajo y capital, el aumento de la inversión conduce a una elevación de los precios que reportará una ampliación de los beneficios. En términos de distribución funcional la participación de los beneficios sobre la renta verá incrementar su nivel, cuanto mayor sea la propensión de los empresarios a consumir e invertir y, por definición, se reducirá la participación de los salarios ${ }^{7}$.

$$
\begin{gathered}
\frac{B}{Y}=\frac{1}{S p-S w} * \frac{I}{Y}-\frac{S w}{S p-S w} \quad \begin{array}{l}
\frac{B}{Y}=\text { Participación beneficios } \\
Y=\text { Producción I I Inversión } \\
\frac{W}{Y}=\text { Participación salarios }
\end{array} \\
1-\frac{B}{Y}=\frac{W}{Y}
\end{gathered}
$$

En consecuencia, de la teoría kaldoriana podemos inferir que un incremento de la inversión y el consumo de los empresarios en el seno de una economía capitalista como la que describe, conduce a un descenso de la participación relativa de los salarios sobre la renta.

Aunque no se enmarque en ninguna de las dos perspectivas anteriormente mencionadas por su carácter sociológico, la obra de los autores franceses J. Marchall y J. Lecaillon La Répartition du revenu national (1958) es muy útil para denotar la importancia que tienen las estructuras de poder a la hora de determinar la participación de los salarios. Estos autores sostienen que la producción es un acto colectivo mientras que el consumo es de carácter individual, por tanto en el nivel productivo pueden existir agentes, fundamentalmente los empresarios, que según la posición que ocupan en la escala social, económica o política sus acciones tienen un efecto directo sobre

\footnotetext{
${ }^{7}$ La teoría de Kaldor fue complementada por Pasinetti en 1962. Para alcanzar los resultados obtenidos Kaldor mantuvo el supuesto de que la propensión a ahorrar de los trabajadores era igual a cero. Pasinetti logró alcanzar los mismos resultados matizando que esta propensión podía no serlo, pero no por ello se modificaba su imparcialidad en el largo plazo. Esto le valió para otorgar mayor generalidad a su modelo.
} 
Baratas, Luis. Distribución funcional de la renta: análisis de la divergencia en la participación salarial entre Alemania y España 1999 - 2013.

otros, por lo general los trabajadores. El poder que cada nivel puede ostentar pasa por: I) las acciones que pueden desarrollar en el interior de las estructuras con la intención de mantener el statu quo, o II) las acciones sobre las estructuras con la intención de transformarlas. De esta manera la evolución de la DFR queda totalmente condicionada a la posición económica, social y política que tienen los actores que participan en la lucha de clases.

A partir de la década de los setenta del siglo XX los análisis teóricos fueron perdiendo presencia en la primera línea académica. No solo porque las grandes figuras de estas corrientes fuesen abandonando sus cátedras, incluso falleciendo, a lo largo de estas décadas (King 2009 pp136); sino también porque en la práctica el conflicto capital-trabajo se fue desvirtuando por varios motivos: la creciente obtención de rentas del capital por parte de los trabajadores, la aparición del autoempleo, el proceso de globalización y la financiarización de la economía mundial. Esto provocó que la literatura académica dejase de preocuparse por renovar o actualizar las viejas teorías de la primera mitad del siglo, y pasarán a investigar de una manera más empírica sobre las causas específicas que ocasionaban las variaciones en la distribución. Desde la década de los noventa comenzaron a florecer multitud de trabajos que trataron de identificar los factores explicativos concretos del descenso continuado de la PS.

Conforme la economía mundial fue tomando un cariz cada vez más neoliberal, algunos trabajos académicos se fueron desligando de la noción de clase que impregnaba las viejas teorías del siglo XX. Trabajos como los de Blanchard y Giavazzi (2003) sustraían cualquier componente social de los mercados de bienes y de trabajo y sostenían un argumento en favor de la eficiencia y el buen funcionamiento en base a la desregulación de los mercados, reconociendo que ésta repercutía negativamente en la PS. En la misma línea el Fondo Monetario Internacional (2007) y la Comisión Europea (2007) identificaron al proceso de globalización, a las innovaciones tecnológicas que de carácter individual pudiesen introducir las empresas y al grado de cualificación de la fuerza de trabajo -conceptualizado como capital humano- como los factores determinantes del descenso de la PS. Estos trabajos excluían del análisis las relaciones de poder que pudiesen tener lugar entre agentes y formulaban, bajo un paradigma ortodoxo, explicaciones de carácter individualista sin especial atención a la dinámica sistémica de funcionamiento.

Por otro lado se conformó una línea de investigación que mantenía la noción de clase en el trasfondo de sus ideas -propio de la economía política- si bien, no del todo homogénea a la hora de dar forma a sus trabajos. Existían y existen quienes conservan una vinculación más directa con algunas de las teorías anteriormente citadas y quienes se alejan de esas construcciones teóricas para realizar trabajos de un marcado carácter empírico.

Respecto a los primeros, hay algunos autores que identifican los cambios en la demanda como un elemento propenso a la reducción de la PS. Esto se produce por medio de la imposición de salarios y condiciones laborales a la baja para ganar competitividad y aumentar los márgenes de beneficio, que provocan que 
Baratas, Luis. Distribución funcional de la renta: análisis de la divergencia en la participación salarial entre Alemania y España 1999 - 2013.

la importancia de las rentas para el consumo sea cada vez menor sobre la composición de la demanda (Bogliciano 2009). Otros autores hacen referencia al patrón de crecimiento en una economía donde no existen normas limitadoras del monopolio, de manera que, una evolución creciente de la inversión y de los precios con la intención de hacer aumentar los crecientes márgenes de beneficio, conducen a un progresivo descenso de la PS (Onaran y Galanis 2012). También Giammarioli, Messina, Steinberger y Strozzi (2002) aúnan sus esfuerzos en conformar una explicación al descenso de la PS en base al papel que juega en el ciclo económico el poder de negociación de los trabajadores. Según el patrón de crecimiento de la economía y el momento del ciclo en el que se encuentre el poder de negociación de los trabajadores puede ser mayor o menor, y por tanto, la PS puede aumentar/reducirse con mayor o menor intensidad. Otras posiciones serían las de Young (2004) y Arpaia, Pérez y Pichelman (2009) con las que tratan de evidenciar cómo según la morfología del tejido productivo entre grandes y pequeñas empresas, y los diferentes grados de utilización de mano de obra entre la industria y los servicios se configura un determinado nivel de poder de negociación que condiciona el reparto de las ganancias de productividad obtenidas entre beneficios y salarios.

Respecto a los segundos cabe destacar una serie de trabajos empíricos donde se contrasta de manera econométrica la incidencia de determinados factores en el descenso de la PS en economías o áreas económicas concretas. En este sentido, uno de los trabajos de referencia es el elaborado por Stockhammer (2013) donde contrasta la incidencia de factores como la globalización, el cambio tecnológico, la financiarización y la reducción del estado de bienestar en el declive de la PS en 71 países (28 desarrollados y 43 subdesarrollados) desde 1970 hasta 2007 . Econométricamente obtiene que los factores que más han contribuido a dicho descenso son la financiarización y la reducción del estado de bienestar. Otro de los trabajos de referencia, muy similar a este en cuanto a la utilización de técnicas econométricas, es el de Ellis y Smith (2007). Ellos concluyen que el factor decisivo ha sido el progresivo deterioro de los sistemas de negociación colectiva en los países OCDE que ha conducido a una reducción del poder de negociación de los sindicatos. En una línea similar la Organización Internacional del Trabajo (2011) llevó a cabo una investigación mediante la cual manifestaban que el buen diseño de un marco regulador de los derechos de los trabajadores, junto con el establecimiento de un apropiado salario mínimo evitaría el descenso permanente de la PS. Por su parte Azmat, Manning y Van Reenen (2007) comprueban la incidencia que tuvo sobre las principales economías desarrolladas la privatización de sus redes industriales, fundamentalmente empresas tecnológicas, de comunicaciones y de servicios públicos. Las privatizaciones generaron situaciones de problemas de agencia y oprimieron la negociación colectiva, de modo que explican buena parte del descenso de la PS. Reed y Latorre (2009) realizaron un análisis de la economía británica desde 1996 a 2007 donde trataban de calcular la incidencia de los procesos migratorios en la PS. Pese a que el impacto resultó no ser demasiado fuerte, es significativa la incidencia que la llegada de inmigrantes dispuestos a asumir salarios inferiores a los nativos tenía sobre el descenso de la PS. En este mismo sentido salarial Karanassou y Sala (2013) recogen el testigo y establecen 
Baratas, Luis. Distribución funcional de la renta: análisis de la divergencia en la participación salarial entre Alemania y España 1999 - 2013.

una relación entre el empeoramiento de la PS en economías con un índice de Gini muy alto. De esta manera sostienen que la fuerte desigualdad salarial es otro factor desencadenante de la pérdida de cuota de los salarios.

Ante las numerosas referencias expuestas es indiscutible la dificultad de encontrar una explicación monocausal a la variación de la PS en las últimas décadas. En este sentido podemos inferir las siguientes conclusiones de este repaso bibliográfico: en primer lugar el rechazo a los postulados neoclásicos fue una premisa de partida que nos ha llevado a buscar explicaciones en otras escuelas más fértiles para ofrecer una explicación sobre nuestra temática. En segundo lugar la revisión de literatura ha evidenciado la necesidad de una concepción social de la problemática de la distribución, entendida como un conflicto social definido por los equilibrios de poder entre las distintas clases sociales. De este modo hemos identificado variables explicativas que no solo tienen relación con la esfera productiva, y aun cuando la tienen, van más allá de la productividad marginal. En este sentido encontramos la inversión, las innovaciones tecnológicas, la composición sectorial y el grado de monopolio de las industrias. También variables de ámbito laboral como el poder de negociación, las instituciones del mercado de trabajo, la cualificación de la mano de obra y las desigualdades salariales; variables referentes a procesos a escala mundial como la globalización, la financiarización y los fenómenos migratorios; y variables de carácter político como las privatizaciones y la reducción del estado de bienestar. Junto a ellas, otras variables difíciles de clasificar tan nítidamente como las propensiones a consumir/ahorrar, las decisiones y contexto monetario y el poder de determinados grupos sociales, políticos y económicos.

Las variables denominadas productivas como la inversión -que se materializa siempre y cuando existan expectativas de beneficio para rentabilizarla-, teorizada por Kaldor y apuntada de manera más empírica por Onaran y Galanis (2012), o las innovaciones tecnológicas en los distintos segmentos industriales y de servicios como señalan Young (2004) y Arpaia et al (2009), derivan en incrementos de productividad cuando dichas actuaciones son lo suficientemente funcionales y beneficiosas con la estructura productiva de cada país. Es decir, el proceso de mecanización inherente a la dinámica capitalista aumenta la productividad y transforma la estructura productiva. A su vez la tendencia decreciente de la tasa de ganancia impulsa al alza la tasa de plusvalía por lo que se reduce la PS. Por otro lado las variables laborales como la existencia de instituciones en el mercado de trabajo eficaces y eficientes -que configuren un marco regulador protector de los derechos de los trabajadores- (OIT, 2011), el grado de cualificación de la mano de obra (FMI y CE, 2007) y los sistemas de negociación colectiva (Ellis y Smith, 2007; Giammarioli et al, 2002; OIT, 2011) redundan en la capacidad que tiene el factor trabajo de beneficiarse de los incrementos de productividad experimentados. Es decir, el poder de negociación para apropiarse de esos incrementos de productividad. Cuando el nivel de sindicalización de una economía es relativamente alto el poder de negociación de la clase trabajadora permite beneficiarse en mayor medida de los aumentos de productividad. Sectorialmente la industria posee un grado de sindicalización mayor que las actividades de servicios, por lo que las economías con un mayor 
Baratas, Luis. Distribución funcional de la renta: análisis de la divergencia en la participación salarial entre Alemania y España 1999 - 2013.

dinamismo industrial pueden experimentar niveles de PS superiores. La desregulación de los mercados de trabajo como señalan Blanchard y Giavazzi (2003) es otra variable destacada pues modifica sustancialmente la naturaleza y el funcionamiento de las relaciones laborales. En muchos casos abre la puerta a nuevas modalidades contractuales entre empleados y empleadores que desequilibran el poder de negociación en favor de los segundos.

En cuanto a los procesos a escala global, la globalización a la que se refieren desde autores convencionales como el Fondo Monetario Internacional y la Comisión Europea hasta otros heterodoxos como Stockhammer (2013) y Bogliciano (2009), obliga a las economías a orientar buena parte de su actividad económica hacia al exterior. De este modo si se especializan en la producción y comercialización de productos de elevada productividad y un alto valor añadido su inserción en este proceso supondrá notables beneficios, en cambio, en una situación opuesta da lugar a fuertes relaciones de dependencia y subordinación económica con respecto a otros países. En el caso de la financiarización (Stockhammer, 2013; Arpaia et al, 2009) ocurre algo similar, ya que la excesiva dimensión de actividades financieras dentro del PIB de un país, caracterizadas por unos bajos niveles de productividad, conduce a un regresión de las actividades productivas y a que los niveles de productividad en general sean menores. Además de a una dependencia de las rentas financieras. De igual modo los fenómenos masivos de migraciones (Reed y Latorre, 2009) conducen a una reducción de la productividad debido a la existencia de un mayor número de trabajadores, junto con una reducción del poder de negociación al estar dispuestos a asumir salarios inferiores. Respecto a las autodenominadas variables políticas, los procesos de privatizaciones mencionados por Azmat et al (2007) y de reducción del Estado de Bienestar como indica Stockhammer (2013), tienen sus consecuencias en términos de productividad y de salarios mediante, por un lado, la contracción de los salarios indirectos y por otro, a través de la primacía de la lógica mercantil en el funcionamiento interno y externo de los sujetos anteriormente controlados por un ente público. Respecto a las variables no encuadradas en esa clasificación, una mayor oferta monetaria produce incentivos para la inversión -siempre y cuando existan buenas expectativas- de modo que la productividad se vería incrementada también por esta vía. Mientras que el poder que pueden llegar a ostentar determinados actores empresariales o sindicales condicionará fuertemente la correlación de fuerzas a la hora de la negociación colectiva.

El efecto que ocasionan todas estas variables indicadas se canaliza sobre la PS mediante dos vías. Por un lado, sobre la variación que puede experimentar la productividad, y/o por otro sobre el reparto que se produce de esas variaciones entre los factores productivos ${ }^{8}$. Si bien podemos extraer de la revisión de literatura la conexión teórica entre las variables explicativas y la variación y reparto de la productividad, nos falta por conocer cómo es la articulación concreta entre ambas dimensiones para los casos de España y Alemania. Por tanto, como paso previo a nuestro análisis empírico, realizaremos una breve particularización de esas conexiones entre las variables explicativas y las dos

\footnotetext{
${ }^{8}$ Véase Anexo 1 como resumen de la estructura teórica que seguimos en el trabajo.
} 
Baratas, Luis. Distribución funcional de la renta: análisis de la divergencia en la participación salarial entre Alemania y España 1999 - 2013.

dimensiones citadas -variación de la productividad y su distribución entre factores- en el contexto específico en el que se encuentran España y Alemania. Versando esta contextualización en los dos elementos que, bajo nuestra opinión, configuran dichas dimensiones, como son la estructura productiva y el régimen de negociación colectiva.

Figura 1. Diagrama resumen de las variables explicativas de la participación salarial.

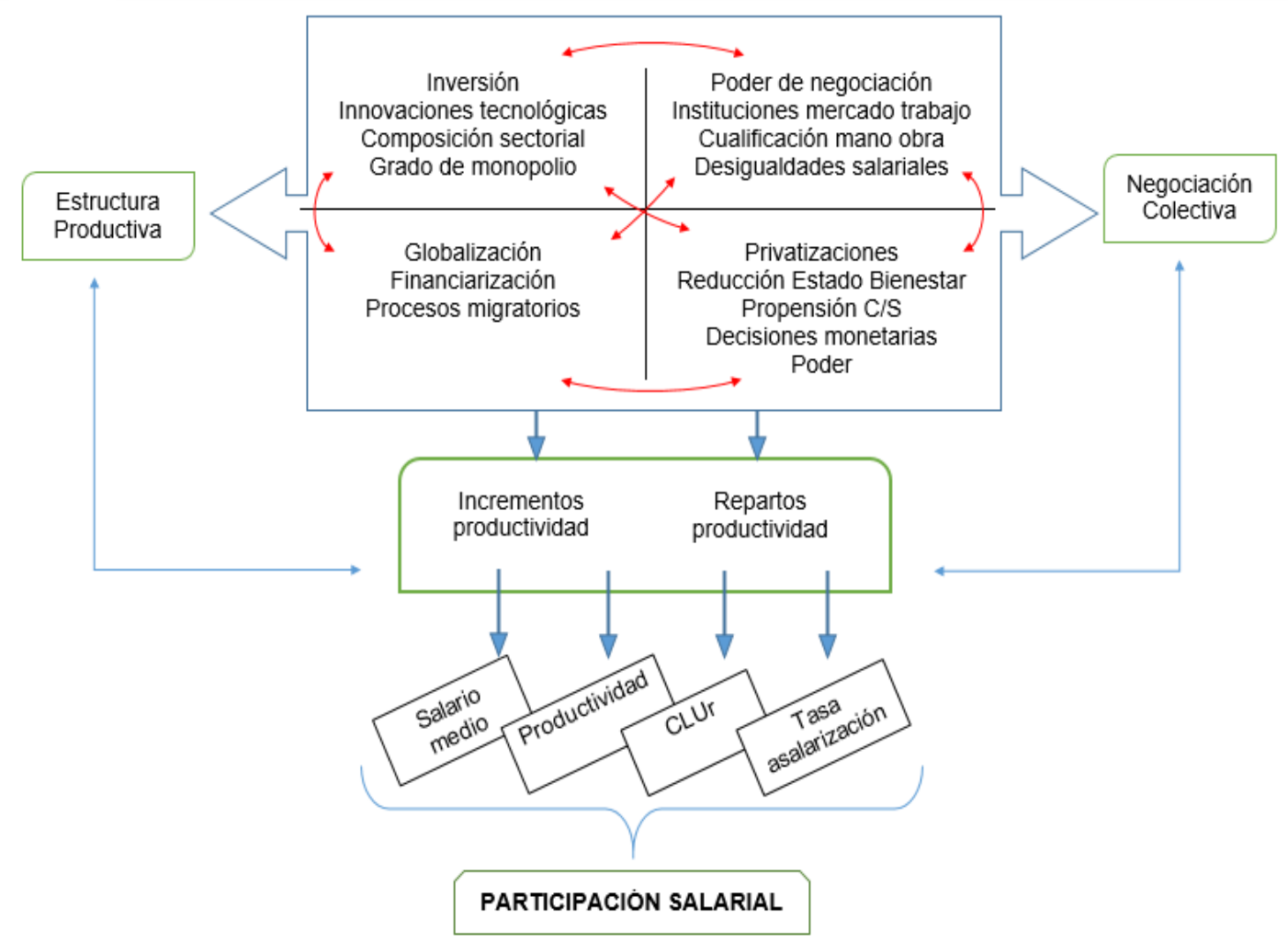

Fuente: elaboración propia.

\subsection{Notas metodológicas}

Antes de llevar a cabo el análisis empírico con el que identificar las raíces de la reciente divergencia en la PS entre España y Alemania también es preciso que realicemos algunas aclaraciones metodológicas. Comenzaremos por identificar y descomponer las magnitudes que conforman la PS. Esta variable se define convencionalmente - de forma analítica como:

$$
\begin{array}{lll} 
& \begin{array}{l}
W=\text { Compensacióntrabajadores } \\
A=\text { Empleo asalariado }
\end{array} & \frac{W}{A}=\text { Salario medio } \\
P S=W / \boldsymbol{Y}=\text { CLUr } * T A=\frac{\boldsymbol{W}}{\frac{\boldsymbol{A}}{\boldsymbol{Y}}} * \frac{\boldsymbol{A}}{\boldsymbol{N}} & \begin{array}{l}
N=\text { Empleo total } \\
\text { CLUr }
\end{array} \quad \frac{W / A}{Y / N}=\text { Coste laboral unitario real }
\end{array}
$$

Papeles de Europa

Vol. 28, Núm. 2 (2015): 54-89 http://dx.doi.org/10.5209/rev_PADE.2015.v28.n2.51903 
Baratas, Luis. Distribución funcional de la renta: análisis de la divergencia en la participación salarial entre Alemania y España 1999 - 2013.

$$
\begin{aligned}
& T A=\frac{A}{N}=\text { Tasa de asalarización } \\
& P S=\frac{W}{Y}=\text { Participación salarial }
\end{aligned}
$$

De esta manera, el devenir de la PS quedará determinado analíticamente por la evolución de: i) el salario medio, ii) la productividad y iii) la tasa de asalarización.

Los datos que se presentarán a continuación han sido extraídos de las bases de datos Eurostat, EU-Klems y Ameco para ser tratados manualmente, de modo que requiere algunas aclaraciones.

La compensación a los trabajadores (W) abarca los sueldos y salarios junto con las cotizaciones sociales y se cuantifica en términos nominales. Para los datos de empleo, tanto asalariado (A) como el empleo total incluyendo a los trabajadores por cuenta propia $(N)$, la unidad en la que se presentan es el equivalente a las horas totales de trabajo. En consecuencia el salario medio hace referencia a la ganancia nominal media por asalariado. EI PIB (Y) está recogido en términos nominales, por tanto, la productividad media por trabajador está presentada igualmente en términos nominales, es decir, incluye los diferenciales de inflación existentes en cada país. En consecuencia la variación que pueda experimentar productividad nominal puede deberse a una variación real de la productividad o a la variación de los precios. Como resultado de dividir estos dos ratios -salario medio y productividad nominal- obtenemos el Coste Laboral Unitario en términos reales (CLUr). Dimensión en la tiene sentido interpretar esta variable cuando hablamos de distribución ya que analíticamente, en sí misma, es el equivalente al peso de la masa salarial en términos nominales sobre la producción nominal y además goza de sentido económico al comparar dos variables bajo una mismo tipo de medición. Tras la ponderación que supone multiplicar el CLUr por la tasa de asalarización de la economía (proporción de trabajo asalariado dentro del trabajo total) obtenemos, de una manera más afinada, la PS. Cuando se produce un aumento (reducción) de los costes salariales por unidad de producto, o bien de la proporción de trabajadores asalariados sobre el total, la PS aumenta (disminuye). De esta forma concluimos obteniendo el peso real de las rentas salariales sobre el producto final de la economía.

Para acotar en el tiempo tanto la interpretación de las tendencias como la posterior explicación causal razonada, revisaremos el desarrollo de las magnitudes anteriormente descompuestas durante el periodo $1999-2013$. A su vez, en este lapso de tiempo diferenciaremos cuatro subperiodos, dos pre-crisis y otros dos post-crisis, justificados por el instante del ciclo económico y el momento histórico en el que se enmarca cada uno. Nuestro análisis los iniciamos en 1999 por ser el momento en el que se establecieron las paridades fijas del euro con respecto las diferentes monedas europeas, y porque supuso el inicio de la puesta en funcionamiento de la misma. Este punto de origen es escogido especialmente porque implicó la oficialización de un determinado marco 
Baratas, Luis. Distribución funcional de la renta: análisis de la divergencia en la participación salarial entre Alemania y España 1999 - 2013.

institucional que provocaría la pérdida efectiva del control sobre el tipo de cambio y condicionaría las políticas monetarias y fiscales de los países europeos. El primer subperiodo lo extendemos hasta 2003. El segundo subperiodo (2004 2007) abarca los últimos años de la fase expansiva del ciclo económico, que se truncaron con el crack financiero. El tercer subperiodo (2008-2010) comprende los años más rudos de la crisis en términos de empleo y PIB mientras que, por último, el cuarto subperiodo (2011 - 2013) compendia los años en los que se puso en marcha la parte más importante del ajuste salarial en el mercado de trabajo, al menos en España.

Las tendencias que se presentarán a continuación corresponden a las economías de España y Alemania a nivel general, aunque si bien, para ganar profundidad en la investigación, hemos extraído los datos según la desagregación estandarizada NACE-2, para un total de 21 ramas, que se irán presentando complementariamente.

\section{CONTEXTUALIZACIÓN ESTRUCTURAL: INTRODUCCIÓN A LA ECONOMÍA POLÍTICA DEL EURO}

La puesta en funcionamiento del euro ha constatado los trascendentales desequilibrios estructurales que padece el área económica europea. La selección del caso de estudio de este trabajo es uno de los mejores ejemplos para clarificar algunos de ellos al tratarse de la economía motriz de la Unión Europea y la economía más importante de la periferia europea, entre las que se ha producido, primero, una relativa similitud en el descenso de la PS, y a continuación una significativa divergencia. En las sucesivas líneas contextualizaremos el estudio de la DFR de Alemania y España en la dinámica regional centrando el interés en las divergencias productivas y salariales que son las relevantes para la DFR, abordando de manera específica la estructura productiva y la negociación colectiva de cada país y las implicaciones que pueden tener sobre la PS.

Las distintas estructuras productivas de los países, no articuladas ni planificadas entre sí, originan uno de los principales desequilibrios que tiene lugar en el seno de la unión monetaria, los desequilibrios productivos. La construcción de una moneda única en economías con distintas escalas productivas, sin la existencia de mecanismos de compensación, ha reforzado las originarias y desequilibradas especializaciones productivas entre unas economías y otras. Tal es así que se ha acabado configurando una estructura productiva y una división del trabajo de carácter dicotómico entre el norte y el sur de Europa (Álvarez et al., 2013). Por un lado los países del norte y centro de Europa, con Alemania a la cabeza, poseen unas estructuras productivas sólidas asentadas, fundamentalmente, en actividades industriales que copan relativamente mayor parte del PIB y del empleo que en los países periféricos. Estas actividades, concentradas en segmentos del mercado con un alto grado de monopolio como la industria energética, química, de telecomunicaciones y de bienes de equipo, han concentrado gran parte de la inversión productiva y se han especializado principalmente en la producción de bienes de alto componente tecnológico. Lo 
Baratas, Luis. Distribución funcional de la renta: análisis de la divergencia en la participación salarial entre Alemania y España 1999 - 2013.

que ha permitido la generación de un alto valor añadido por unidad y altos niveles de productividad real.

En Alemania, desde 1997 a 2007, las ramas industriales de mayor intensidad tecnológica fueron las que más crecieron del conjunto de la industria, aumentando fuertemente su productividad y su peso sobre el total de empleo del sector (Garzón, 2014). Los aumentos de productividad fueron logrados esencialmente por innovaciones y mejoras reales en el proceso productivo que dinamizaban el crecimiento de la producción. El alto componente tecnológico y la buena percepción de este tipo de productos por un lado, junto con la apreciación de la moneda europea en el concierto internacional por otro, han permitido a Alemania adoptar una determinada estrategia comercial con las que insertarse en el proceso de globalización de manera dominante. La gran competitividad de sus exportaciones ha reportado voluminosos superávits a la economía alemana que ha permitido financiar las importaciones de otros países europeos. Estos superávits comerciales han aumentado progresivamente desde un $3,18 \%$ del PIB en 1999 hasta un 7,07\% en 2014, pasando de una tasa de cobertura del $114,66 \%$ a un $124,11 \%$ en ese mismo lapso de tiempo.

En el otro extremo se encuentran las economías periféricas del este de Europa y, sobretodo, del eje Mediterráneo particularmente España. Esta economía se caracteriza por una endémica debilidad industrial y una sobrevaloración de actividades terciarias como el turismo y la construcción. Su estructura productiva industrial es mucho más débil y eso se ve reflejado en sus limitados resortes tecnológicos, financieros e incluso laborales (Álvarez et al, 2013). Las actividades que copan una mayor parte del PIB y concentran gran parte de las inversiones son, esencialmente, los servicios turísticos, inmobiliarios, comerciales y financieros; mientras que se relega a un segundo plano a las actividades industriales. Estas actividades industriales están localizadas en segmentos de media y baja tecnología de modo que reportan un menor valor añadido y además gozan de niveles de productividad muy bajos. Durante estas últimas décadas la productividad real en la economía española ha permanecido estancada y los incrementos experimentados han sido ocasionados, fundamentalmente, por la evolución de los precios, que llega a explicar en algunos momentos más del $90 \%$ de la variación de la productividad nominal. Un incremento de los precios en consonancia con la evolución exponencial de los precios de la vivienda. La euforia de la burbuja inmobiliaria arrastró al conjunto de la economía española hacia un modelo abocado al colapso (Rodríguez \& López, 2011).

Esta morfología industrial unida a la evolución del tipo de cambio del euro, perjudicial mientras se producen apreciaciones, solo deja margen para una estrategia comercial fuertemente deficitaria que se convierte en crónica en tanto que estos países no son capaces de superar estas limitaciones. El déficit comercial español es una característica intrínseca de la economía que oscila entre el $2 \%$ en sus momentos más reducidos y el $10 \%$ en sus momentos más álgidos. La historia ha demostrado que España solo logra suavizarlo en fases de notorio descenso de las importaciones más que por un mayor dinamismo de las 
Baratas, Luis. Distribución funcional de la renta: análisis de la divergencia en la participación salarial entre Alemania y España 1999 - 2013.

exportaciones en valor y/o volumen. La relativamente baja tasa de cobertura, en torno al $90 \%$ en 2013, nos da muestras de la débil competitividad exportadora del país. Ante la imposibilidad de aplicar devaluaciones externas (monetarias), con la intención de que sus exportaciones ganen competitividad, España y otros países periféricos, como Portugal y Grecia, han optado por aplicar devaluaciones internas (salariales). Estas reducciones efectivas de los costes laborales unitarios tienen un alcance muy limitado como estrategia competitiva ya que ignoran los determinantes estructurales de la competitividad, precisamente los que tienen que ver con la estructura productiva. A la par, tiene consecuencias muy negativas en otras dimensiones como el consumo, la distribución de la renta, etc. (Felipe \& Kumar, 2011).

Precisamente otro de los principales desequilibrios que existen dentro de la zona euro son los desequilibrios sociales ocasionados, en buena medida, especialmente en estos últimos años, por las reformas aplicadas para poder materializar unas determinadas estrategias competitivas. Estas estrategias competitivas necesitaron de reformas laborales cuyos resultados han dejado patente la no corrección de los desequilibrios productivos y el surgimiento de distintos patrones salariales a seguir por los países europeos. Cada vez es más evidente la brecha salarial que se produce entre las economías del centro y las periféricas. En el año 2013 en Alemania cada trabajador dedicó 1.388 horas de trabajo por un salario medio anual de 44.700€. Sin embargo, en España, la media total de horas dedicadas al trabajo es de 1.665 horas por trabajador/año, a cambio de un salario medio anual de 26.027€. Es decir, de media, en la periferia europea se trabajan más horas con niveles salariales inferiores. En la evolución de la dinámica salarial han jugado un papel muy importante las reformas estructurales aplicadas en los mercados de trabajo y en particular las que afectaron al poder de negociación (Pérez Infante, 2012). Estas reformas ya fueron implementadas en países como Alemania antes de la llegada de la crisis de 2008 y han sido exigidas en los países periféricos como contrapartida a la ayuda financiera europea para paliar sus manifestaciones de crisis.

En el caso alemán las conocidas como Leyes Hartz III y IV, aprobadas en 2003, supusieron un drástico cambio en el marco regulador del mercado laboral existente hasta entonces y el inicio de una etapa de descenso de los salarios y los costes laborales unitarios más intenso que en otros países como España (Bilbao y Ochando, 2012). Afectó particularmente al régimen de prestaciones por desempleo y de costes de despido, ya que se consideraban dos elementos susceptibles de ralentizar tanto el proceso de búsqueda de un nuevo empleo, como la adaptación de las empresas a las necesidades del contexto económico. También afectó muy incisivamente al poder de negociación de los trabajadores sobre los salarios y el resto de condiciones laborales, primando las relaciones bilaterales entre trabajadores y empresas sobre el mecanismo de negociación colectiva canalizado por los sindicatos (Bispinck, \& Schulten, 2012). En el año 2003 comenzó una etapa de moderación de las compensaciones a los trabajadores que ocasionó un estancamiento del salario relativo. Al igual sentó las bases para que el establecimiento de los incrementos salariales anuales se comenzase a realizar, no conforme a la evolución de la inflación -y por tanto 
Baratas, Luis. Distribución funcional de la renta: análisis de la divergencia en la participación salarial entre Alemania y España 1999 - 2013.

mantuviese un determinado poder adquisitivo-, sino conforme a los incrementos de productividad. El consecuente desfase entre el crecimiento de la productividad y del salario relativo, reflejo de la correlación de fuerzas del momento entre el factor trabajo y capital, propició un creciente descenso de los costes laborales hasta la llegada de la crisis.

Como veremos detalladamente en el siguiente apartado, en España el modelo productivo instaurado dio lugar a una etapa de euforia en los precios que condujo a un fuerte crecimiento de las compensaciones nominales a los trabajadores y de la productividad. Dado que la correlación de fuerzas era igualmente débil para el factor trabajo, los incrementos del salario relativo se situaron por debajo de los incrementos nominales de la productividad. Esto provocó un leve descenso de los costes laborales unitarios. La estrategia de reducción de costes laborales unitarios en búsqueda de una mayor competitividad se volvió más explícita a partir de 2010 cuando se empezaron a aplicar los ajustes salariales. Junto a esta evolución, las reformas laborales de 2010 y 2012, muy similares a las alemanas, han terminado por configurar una dinámica salarial regresiva muy desfavorable para los intereses de la clase trabajadora.

La pérdida de relevancia de la negociación colectiva en el establecimiento de los salarios ha sido uno de los desencadenantes de esa reducción. En el año 2000 el número de trabajadores sujetos a convenio colectivo era de 7,14 millones, mientras que para el año 2012 ese número se ha reducido a los 4,36 millones de trabajadora. En esta dinámica de reducción también se han visto afectados tanto el número de convenios efectivos como el de empresas suscriben estos convenios (Pérez Infante, 2012). Las sucesivas reformas laborales han posibilitado la utilización de elementos como la cláusula de descuelgue salarial que permite la inaplicación de convenios colectivos de manera unilateral por parte de la empresa-, así como el intento de aminorar el papel de los sindicatos en las relaciones empresariales y/o sectoriales, ya de por si maltrecho por la inserción en el proceso de globalización de la economía española (Köhler \& Calleja, 2008).

Además estas reformas laborales han posibilitado nuevas formas de contratación individualizada o por medio de empresas de trabajo temporal. Estas contrataciones más beligerantes con los derechos laborales se manifiestan bajo la figura de trabajadores por cuenta propia que desarrollan su labor por y para la empresa contratante -falsos autónomos-. Si añadimos que la evolución de los precios en España era superior a la de los crecimientos salariales, nos encontramos con un estancamiento de las compensaciones reales que reducían el poder de consumo de los asalariados. Este solo se pudo mantener gracias al endeudamiento, lo que fraguó otro de los grandes problemas de las economías periféricas, la deuda.

De esta manera se ha tejido una estrategia de contención salarial que ha provocado: 1) en términos estrictamente económicos, una reducción de la demanda interna a través de la depresión del consumo que ha arrastrado a su vez a una reducción de los beneficios y por tanto de la inversión. Todo ello 
Baratas, Luis. Distribución funcional de la renta: análisis de la divergencia en la participación salarial entre Alemania y España 1999 - 2013.

acompañado de un fuerte proceso de endeudamiento del sector privado. (Álvarez et al, 2013). 2) En términos sociales, un modelo generador de empleo en sectores de baja productividad y bajos salarios que da lugar a un fuerte proceso de incremento de la desigualdad de la renta, tanto personal como factorial (Bilbao y Ochando, 2012).

Vistas estas dos dimensiones que consideramos esenciales para profundizar en nuestro análisis empírico, resulta interesante preguntarse acerca de la posible relación existente entre las estrategias competitivas seguidas por estos países y el nivel -y variación- de sus participaciones salariales. ¿Podemos atribuir algún tipo de relación causa-efecto entre las primeras para con las segundas? Parece coherente pensar que la especialización en ramas de alto valor añadido, fundamentalmente industriales, puede traer consigo mayores niveles de PS, como es el caso de Alemania.

La labor de dar una respuesta rotunda a las preguntas formuladas no es uno de los objetivos de este trabajo, por lo que lo dejamos abierto a discusión y a futuros trabajos que prueben empíricamente esta relación. Con estas líneas hemos tratado únicamente de contextualizar de manera estructural la situación en la que se encuentra nuestro objeto de estudio. A continuación, sin tratar de llevar a cabo una contrastación en sentido estricto de las hipótesis sobre las causas de la divergencia de las participaciones salariales, llevaremos a cabo un primer acercamiento al análisis pormenorizado de las tendencias seguidas por los componentes que explican la PS.

\section{ANÁLISIS EMPÍRICO DE LA PS Y SUS COMPONENTES EN ALEMANIA Y ESPAÑA, 1999-2013}

Este apartado pretende mostrar las tendencias generales seguidas por la PS y sus respectivos componentes en la economía española y alemana, con la intención de identificar los hechos estilizados que marcan la pauta en el descenso de la PS y la dimensión en la que se encuadran. Observando los resultados de la PS mostrados en el gráfico 1 nos encontramos con que las economías alemana y española han experimentado una evolución análoga de descenso simultáneo en la cuota de los salarios sobre la renta hasta la llegada de la crisis, y otra marcadamente opuesta desde 2010 en adelante.

Desde los años ochenta la PS en estas economías venía experimentando una progresiva pérdida que se prolongará ininterrumpidamente hasta la llegada de la crisis en 2008. Durante los primeros 10 años de nuestro periodo de estudio (1999-2008) España sufrió un descenso absoluto de 4 puntos porcentuales (pp en adelante) pasando del $51 \%$ al $47 \%$. Mientras que en Alemania fue de 5 pp, desde el $54 \%$ hasta el $49 \%$. Con el impacto de la crisis financiera la participación de los salarios experimentó un incremento, de nuevo simultaneo, pero transitorio, en ambos países fruto del efecto composición que tiene sobre nuestra variable las consecuencias negativas que generan las crisis por el lado de la producción y del empleo. Cuando empiezan a aparecer los efectos en términos laborales, algo más rezagados que en términos de PIB, la destrucción de empleo se 
Baratas, Luis. Distribución funcional de la renta: análisis de la divergencia en la participación salarial entre Alemania y España 1999 - 2013.

concentra primero en las actividades de bajos salarios y baja productividad. Generalmente las que gozan de unos menores niveles de PS, por lo que al reducirse los valores inferiores del conjunto se aumenta el promedio de los ratios. Este efecto composición recolocó la PS en el $50 \%$ para el caso de España y en el $52 \%$ para el caso de Alemania.

Una vez diluido el efecto estadístico la divergencia comienza a ser bastante llamativa, puesto que la PS en Alemania a partir del año 2010 parece estabilizarse en torno al 51-52\%, mientras que en España no cesa de decrecer desde ese mismo momento hasta el final de nuestra serie, 45,5\% en 2013. Es decir, tras la crisis la evolución de la PS en ambos países comienza a divergir claramente frente al mantenimiento de una evolución muy similar en los 10 años anteriores. A nivel agregado para España, las ramas que mayor caída de la PS han sufrido para el conjunto del periodo han sido: construcción (45pp), alojamiento y hostelería (44pp), actividades profesionales y servicios a empresas (27pp) y comercio al por mayor/menor (18pp). Por el lado alemán fueron las ramas de: agricultura y pesca (77pp), extractivas (67pp), alojamiento y hostelería (34pp) y construcción (29pp).

\subsection{Productividad del trabajo}

La evolución experimentada por la productividad es consecuencia de la conjunción entre las evoluciones del PIB en términos nominales y del equivalente en horas de trabajo del nivel de empleo total. De esta manera estamos incluyendo en la medición de la productividad el efecto que pueda ocasionar la variación de los precios en cada economía, de tal forma que aunque lo omitamos, en realidad nos referiremos permanentemente a la productividad nominal. Hemos de indicar que anticipamos la exposición de la productividad a la del salario medio porque, bajo nuestra opinión, la evolución de los salarios en una economía está previamente condicionada, entre otras cosas, por la estructura productiva de la misma, donde la productividad del trabajo juega un papel muy sustancial.

La productividad en ambos países viene siguiendo una tendencia creciente desde 1999 que se prolongó hasta 2010, donde los incrementos interanuales españoles fueron superiores a los alemanes, en buena medida gracias a un crecimiento más intenso de los precios. A partir de 2010 la productividad española se estanca mientras que la alemana experimenta variaciones positivas.

Durante el primer periodo de la etapa pre-crisis, 1999-2003, la productividad española creció a razón de un 4,67\% interanual frente al 2,63\% alemán. Este crecimiento para el caso español se explica fundamentalmente por un crecimiento medio interanual de la producción a en términos nominales $(7,80 \%)$ superior al del empleo total (2,99\%). En Alemania el crecimiento del PIBpm no fue tan intenso $(1,80 \%)$ pero se combinó con un leve descenso del empleo total $(-0,81 \%)$ que contribuyó favorablemente a la evolución de la productividad. Durante el segundo periodo, 2004-2007, la productividad en ambos países se comportó de manera similar al primer periodo, a base de crecimientos medios 
Baratas, Luis. Distribución funcional de la renta: análisis de la divergencia en la participación salarial entre Alemania y España 1999 - 2013.

del $4,80 \%$ para España y del $2,76 \%$ para Alemania. Igualmente los comportamientos de la producción y el empleo fueron similares a los del primer periodo en cada país. En España la producción continuó creciendo a un 7,69\% de media interanual y el empleo total a un $2,76 \%$. Mientras que en Alemania el crecimiento de la producción fue de un 3,13\% interanual y el empleo total experimento un leve aumento del $0,36 \%$ de media.

Es preciso señalar el destacado papel que jugó durante esta etapa de expansión económica la evolución de los precios en España. Los incrementos medios interanuales del $4,14 \%$ durante este periodo amplificaron el crecimiento de la producción en términos nominales y por tanto, también del ratio de productividad. Si descontamos el efecto de la inflación y nos quedamos con la productividad en términos reales, su evolución se caracteriza por un estancamiento permanente durante estos años, 0,53\% interanual. En cambio en Alemania la inflación jugo un papel mucho más comedido. Su TVMA se situó en el $0,54 \%$ de modo que su incidencia sobre la variación de la producción y la productividad en términos nominales fue significativamente menor. Los incrementos de productividad durante todo este periodo para el caso alemán no están tan condicionadas por los precios como para el caso español, de ahí el crecimiento medio interanual del $2,16 \%$ de la productividad real alemana.

Durante esta etapa de continuado aumento de la productividad nominal, los sectores que mayores incrementos absolutos experimentaron en la economía española fueron: actividades financieras (90pp), manufacturas (60pp), construcción (60pp), comercio al por mayor/menor (53pp) e industria extractiva (43pp). En Alemania fueron las ramas correspondientes a: industria extractiva (70pp), electricidad, gas y agua (49pp), manufacturas (40pp), actividades inmobiliarias (32pp) y comercio al por mayor/menor (30pp).

Durante el tercer periodo en plena recesión económica, 2008-2010, la reducción del empleo marca la pauta de comportamiento de la productividad para España. Durante todo el subperiodo la fuerte destrucción de empleo $(-2,73 \%)$ superó la contracción del PIBpm $(-0,20 \%)$ de manera que condujo a unos incrementos medios interanuales de la productividad del (2,60\%). En Alemania las contracciones del PIB y del empleo se concentraron en el año 2009 y se compensaron relativamente rápido. De modo que la economía alemana logró mantener tasas tímidamente positivas de productividad $(0,73 \%)$ gracias a un moderado incremento de la producción $(0,98)$ superior al del empleo total $(0,21 \%)$. Conforme avanzan los años España cae en una segunda recesión y no consigue recuperar tasas positivas de creación de empleo. Para este lapso de tiempo, que va desde 2010 hasta 2013, la variación del empleo total y de la producción es de $-0,94 \%$ y $-0,72 \%$ respectivamente; lo que conduce a un estancamiento de la productividad con una variación media anual del apenas $0,22 \%$. Por su parte Alemania recupera tasas positivas del crecimiento del PIBpm $(3,15 \%)$ y del empleo $(0,64 \%)$, que la conducen a incrementos de productividad del $2,48 \%$. Durante toda esta fase el patrón evolutivo de la inflación ha sido similar al de la fase pre-crisis, donde los niveles de precios y las variaciones interanuales fueron superiores en España. Si bien, en este lapso la 
Baratas, Luis. Distribución funcional de la renta: análisis de la divergencia en la participación salarial entre Alemania y España 1999 - 2013.

incidencia de los precios en la productividad de la economía alemana ha sido manifiestamente mayor que antes. Frente a un ligero agotamiento de la productividad real, Alemania consigue leves incrementos de la productividad nominal gracias a la incidencia de ellos.

Gráfico 2. PIBpm(Y), empleo total $\left(L^{*}\right)$ y productividad (Y/L*); 1999-2013

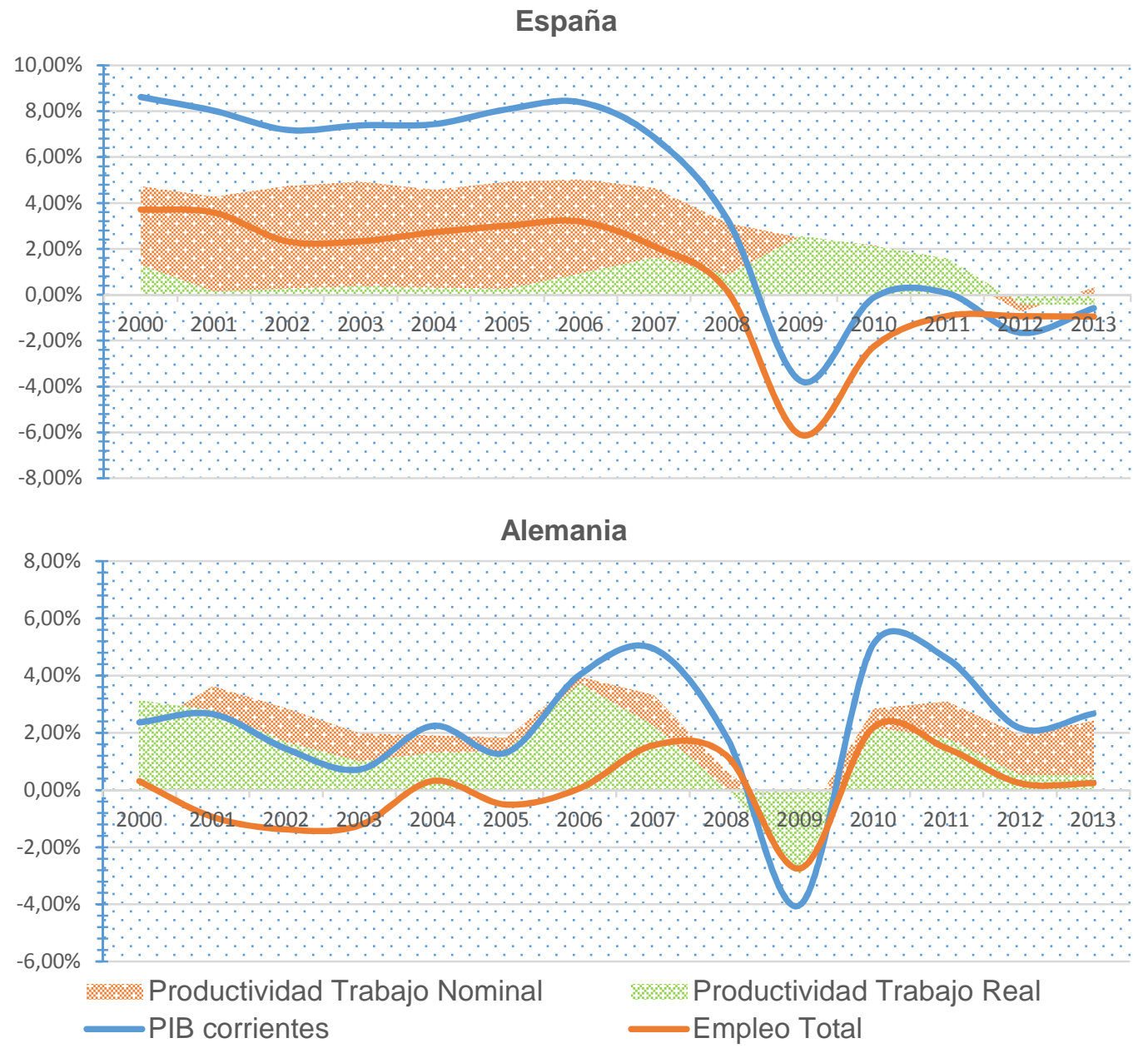

Fuente: elaboración propia.

En la etapa pre-crisis España consiguió aumentar la productividad gracias a que las variaciones de precios que incrementaban artificialmente el crecimiento del PIB en términos nominales lo lograron situar por encima de la generación de empleo. Desde el impacto de la crisis hasta ahora, la productividad permanece estancada por la incapacidad de la economía española de generar suficientes incrementos del PIB, cuando la evolución de los precios ya no supone una propulsión, a pesar de la caída del empleo. En Alemania durante la etapa previa a la crisis los aumentos de productividad se lograron gracias a incrementos reales sin una incidencia tan relevante de los precios. Una vez se corrobora el fin de la recesión en Alemania la productividad experimenta variaciones positivas debido a una recuperación de la producción y un relativo estancamiento del empleo. En estos últimos años la dinámica de precios favorece al desenvolvimiento de la productividad. 
Baratas, Luis. Distribución funcional de la renta: análisis de la divergencia en la participación salarial entre Alemania y España 1999 - 2013.

La evolución de la productividad juega un papel muy importante en la tendencia de la PS, pero para una completa interpretación es necesario ponerla en línea con la evolución del salario medio.

\subsection{Salario medio}

La evolución del salario medio es el resultado de la interacción entre la variación de la compensación a los empleados y la variación del empleo asalariado. Su progreso en ambos países ha sido bastante similar en la trayectoria, pero desigual en los ritmos hasta el estallido de la crisis. En la etapa pre-crisis el salario medio creció tanto en Alemania como en España, si bien con más intensidad en este último. Durante el periodo de crisis la fuerte destrucción de empleo, primero en sectores con peores condiciones laborales e inferiores salarios, condujo a aumentar de manera artificial esta ratio. En 2010, cuando el salario medio se estanca, tiene lugar un punto de inflexión a partir del cual esta variable tomó sendas diferentes en cada país. Mientras que en Alemania recuperó la tendencia alcista, en España se consolidó una tendencia fuertemente decreciente que constata la divergencia en las trayectorias.

En el primero de nuestros subperiodos ,1999 - 2003, el salario medio español crece a una tasa de variación media anual (TVMA) del 3,55\% frente al 2,43\% alemán. Este crecimiento está conducido por un incremento de la compensación a los empleados superior a las variaciones del empleo asalariado. Para el caso de España la relación es de un crecimiento de la compensación a los empleados del $7,18 \%$ TVMA frente a un 3,5\% del empleo asalariado. Por el lado alemán esta relación es de un $1,61 \%$ frente a un $-0,8 \%$. De manera que la diferencia en los ritmos de crecimiento del salario medio está marcada por un notable incremento de la compensación a los empleados en España.

Para el segundo de nuestros subperiodos, 2004 - 2007, la distancia entre el ritmo de crecimiento español y el alemán se dilata. El crecimiento interanual del salario medio español es del $4,11 \%$, mientras que el alemán apenas crece un $0,65 \%$. Las raíces del crecimiento en España vuelven a situarse en el fuerte incremento de la compensación a los empleados $(7,38 \%)$, superior al incremento del empleo asalariado (3,14\%); mientras que en Alemania el estancamiento se debe fundamentalmente a un leve crecimiento de la compensación a los empleados $(0,93 \%)$ y una modestísima recuperación del empleo asalariado $(0,27 \%)$.

Las ramas de la economía española que mayor incremento del salario medio experimentaron en la etapa pre-crisis fueron las de: transporte y almacenaje (71pp), comercio al por mayor/menor (61\%pp), administración pública y defensa (50pp) y manufacturas (48pp). Cabría esperar que el sector de la construcción fuese uno de los más dinámicos en el incremento del salario medio, debido a la alta participación de este sector en el modelo productivo español, pero los incrementos del empleo superiores a los ya de por si elevados incrementos en la compensación a los empleados provocaron que la variación final no fuese de las más significativas (10pp). En Alemania las ramas con un crecimiento más intenso del salario medio - dentro de su modestia- fueron las de: actividades 
Baratas, Luis. Distribución funcional de la renta: análisis de la divergencia en la participación salarial entre Alemania y España 1999 - 2013.

financieras y de seguros (29pp), información y comunicación (25pp), construcción (22pp), electricidad gas y agua (20pp) y manufacturas (20pp).

El periodo 2008 - 2010 es algo particular, pues comienza determinado por el aumento vía efecto composición de la crisis en términos de empleo y culmina con una contracción que sirve como punto de inflexión para dar comienzo a la tendencia divergente. En este lapso de tiempo el salario medio español se incrementa notablemente como resultado del efecto estadístico $(3,57 \%)$ fruto de la fuerte reducción del empleo asalariado (-2,71\%) y el estancamiento de la compensación a los empleados $(0,78 \%)^{9}$. En cambio, en Alemania, se recuperaron incrementos relativamente intensos de la compensación a los empleados (2,25\%). La destrucción de empleo fue menor y se concentró en el año 2009, lo que anuló los incrementos del ratio de asalariados sobre el total que tuvieron lugar en 2008 y 2010 . Esto condujo a una variación apenas existente en este subperiodo $(0,28 \%)$. El resultado de esta conjunción fue un incremento del salario medio del $2 \%$ en Alemania, no tan artificial como había sido en España. En el año 2010 podemos identificar un punto de inflexión desde el cual los salarios medios de ambos países adoptarán trayectorias opuestas.

Como hemos indicado previamente, el cuarto subperiodo desde 2010 hasta 2013 se caracteriza por la evolución divergente del salario medio. Su variación media interanual en Alemania conduce a incrementos del 2,74\% anuales, mientras que en España a reducciones del 2,53\%. El destacado incremento del salario medio alemán contrasta con la fuerte reducción que tiene lugar en España. Este descenso es una de las fuerzas que conduce a la caída de la PS en España. Las raíces de ese crecimiento en Alemania las encontramos en los notables incrementos de la compensación a los empleados $(3,70 \%)$ superiores a la modesta creación de empleo asalariado $(0,93 \%)$. Por otra parte, la reducción del salario medio español en esta última fase es resultado de la fuerte contracción interanual de la compensación a los empleados $(-3,26 \%)$. La persistente reducción del empleo asalariado $(-0,75 \%)$ aminora levemente el ritmo de decrecimiento del salario medio español.

En este último periodo, durante el cual se constata la divergencia en la evolución de los salarios medios, las ramas de la economía española que más ven reducir esta ratio son: actividades inmobiliarias (62pp), construcción (38pp), comercio al por mayor/menor (31pp), alojamiento y hostelería (31pp). Por el contrario las ramas alemanas que experimentan un mayor crecimiento son: administración pública (10pp), información y comunicación (9pp), actividades artísticas, recreación y entretenimiento (8pp), actividades profesionales y servicios a empresa (7pp) y alojamiento y restauración (6pp).

\footnotetext{
${ }^{9}$ El estancamiento durante este subperiodo de la compensación a los empleados es resultado de la anulación del fuerte incremento de 2008 por parte de las sucesivas reducciones de 2009 y 2010.
} 
Baratas, Luis. Distribución funcional de la renta: análisis de la divergencia en la participación salarial entre Alemania y España 1999 - 2013.

\section{Gráfico 3. Compensación empleados (W), empleo asalariado(L) y salario medio}

(W/L); 1999-2013

España

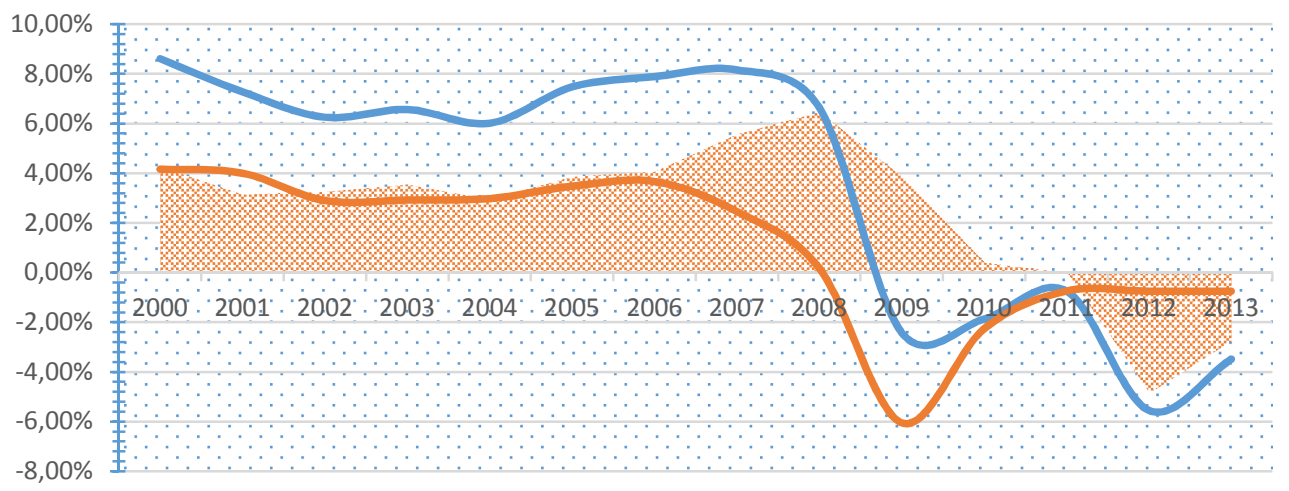

Alemania

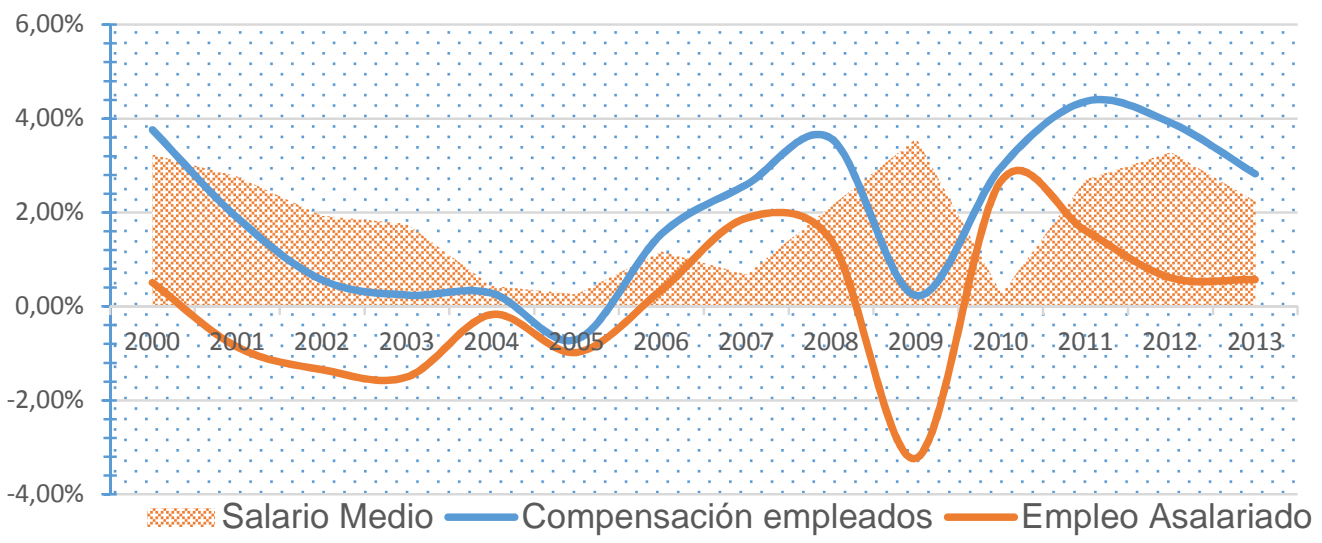

Fuente: elaboración propia a partir de Eurostat.

Por tanto debemos destacar el categórico papel que juega en la variación del salario unitario en España el fuerte incremento de la compensación a los empleados en la etapa pre-crisis. Su posterior contracción en la etapa post-crisis es igualmente decisiva para que el salario medio siga una tendencia a la baja. Por su parte, Alemania incrementa en la etapa pre-crisis levemente su salario medio gracias a una reducción del empleo asalariado en un contexto de moderación salarial. La evolución del salario medio alemán en la etapa postcrisis está totalmente condicionada por la dinamización de las tasas de crecimiento de la compensación a los empleados.

La relación existente entre el salario medio recibido por cada trabajador y la cantidad producida por cada uno de ellos está convencionalmente conceptualizada como el coste laboral unitario, es decir los costes salariales necesarios por cada unidad producida. Es fundamental analizar su evolución para entender el comportamiento de la PS. 
Baratas, Luis. Distribución funcional de la renta: análisis de la divergencia en la participación salarial entre Alemania y España 1999 - 2013.

\subsection{Coste Laboral Unitario real}

La evolución experimentada por el CLU real es el resultado de la interacción entre las variaciones del salario medio y la productividad. El CLU real es frecuentemente utilizado como un indicador explicativo de la distribución factorial, ya que se considera como un equivalente analítico de la participación de los salarios sobre el nivel de renta. Durante la fase de expansión económica previa a la crisis los CLU alemanes y españoles sufrieron un descenso permanente que culmina en el año 2010. Tras el incremento momentáneo que ocasiona la crisis, los CLU reales en Alemania recuperan los niveles precedentes y se estabilizan, mientras que en España retoman una trayectoria descendente.

Durante la etapa previa a la crisis, 1999 - 2007 los CLUr en ambos países siguieron una trayectoria descendiente debido a que los incrementos de la productividad nominal fueron superiores a los incrementos del salario medio. Durante el primer subperiodo la variación de la productividad española $(4,67 \%)$, condicionada fuertemente por la evolución de los precios que explica 4,14 puntos porcentuales de dicha subida, fue superior a la del salario medio $(3,55 \%)$, lo que derivó en un descenso del CLUr de $(-1,07 \%)$. En el caso alemán el descenso del CLUr fue bastante más suave $(-0,19 \%)$ a razón de una diferencia entre variación de la productividad $(2,63 \%)$ y del salario medio $(2,43 \%)$ mucho menor. En este caso la incidencia de los precios solo explica 0,47 puntos de subida. Durante el segundo subperiodo el descenso de los CLUr español fue menor que el alemán. En España descendieron de media un 0,66\%, fruto de una estrecha diferencia entre los intensos incrementos de la productividad $(4,80 \%)$ y del salario medio (4,11\%). Nuevamente 4 puntos porcentuales del aumento de la productividad se deben al aumento de los precios. Para Alemania el descenso fue más intenso de media interanual $(-2,05 \%)$ debido a la diferencia entre los incrementos de productividad (2,76\%), reiteradamente solo inducido por 0,6 puntos de aumento de los precios, y el estancamiento del salario medio $(0,65 \%)$. De esta manera estamos evidenciando el intenso papel redistributivo que juega la inflación en la economía española, en tanto que convierte la distribución funcional de la renta más regresiva para el factor trabajo cuanto peor se ajustan los salarios pactados a la inflación real.

A lo largo de esta fase expansiva las actividades de la economía española que padecieron un mayor descenso de los CLUr fueron: alojamiento y hostelería (39pp), construcción (31pp), industria extractiva (30pp), actividades financieras (25pp) y actividades profesionales y servicios a empresas (17pp). En la economía alemana los mayores descensos se localizaron en: industria extractiva (37pp), actividades inmobiliarias (23pp), alojamiento y restauración (20pp) y suministros de electricidad, gas y agua (19pp).

En la primera fase de la etapa pos-crisis (2008-2010) el significativo aumento del salario medio, fruto del efecto composición mencionado anteriormente, lideró el aumento del CLUr en las dos economías. El aumento fue más destacado en Alemania $(1,32 \%)$ que en España $(0,94 \%)$ gracias al menor ritmo de crecimiento de la productividad en Alemania $(0,73 \%)$ que en España $(2,60 \%)$ durante este 
Baratas, Luis. Distribución funcional de la renta: análisis de la divergencia en la participación salarial entre Alemania y España 1999 - 2013.

subperiodo. En la segunda fase de la etapa pos-crisis (2010-2013) se fragua la divergencia. Mientras que Alemania consigue mantener tasas de variación media anual modestamente positivas ${ }^{10}(0,26 \%)$ gracias a que el salario medio crece ligeramente más que la productividad ( $2,74 \%$ frente 2,48\%), España retoma una trayectoria descendente $(-2,75 \%)$ debido a la notable reducción del salario medio y al estancamiento de la productividad $(-2,53 \%$ y $0,22 \%)$.

Las ramas de la economía española que mayor descenso acumulado del CLU real sufrieron durante este último subperiodo fueron: actividades inmobiliarias (57pp), transporte y almacenaje (38pp), construcción (35pp), comercio al por mayor/menor (32pp) y alojamiento y hostelería (31pp).En Alemania la estabilización del CLU para el total de la economía contrasta con los incrementos acumulados en las ramas de: actividades financieras (19pp) e información y comunicación (9pp); y las reducciones en: comercio al por mayor/menor (12pp) e industrias extractivas (11pp).

Gráfico 4. Salario medio (W/L), productividad trabajo (Y/L') y CLUreal; 1999-2013

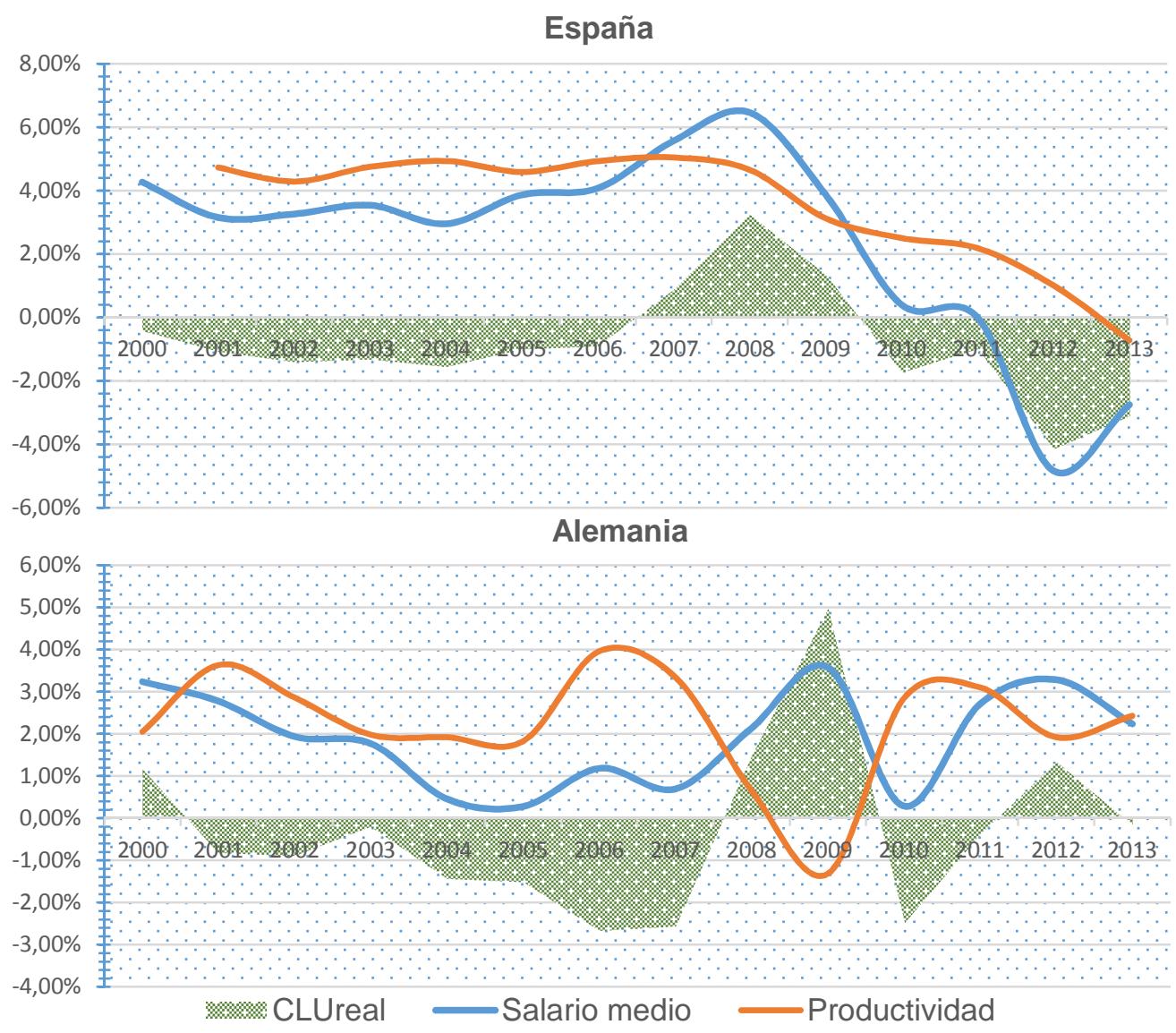

Fuente: elaboración propia a partir de Eurostat.

\footnotetext{
${ }^{10}$ En este subperiodo se produce una oscilación equivalente en ambas direcciones que es lo que produce el estancamiento como media del periodo. Obsérvese gráfico 3.4 años 2010-2013.
}

Papeles de Europa

Vol. 28, Núm. 2 (2015): 54-89 http://dx.doi.org/10.5209/rev_PADE.2015.v28.n2.51903 
Baratas, Luis. Distribución funcional de la renta: análisis de la divergencia en la participación salarial entre Alemania y España 1999 - 2013.

Para terminar de pulir esta identificación de tendencias que nos ayuden a esclarecer los orígenes de la divergencia entre la PS de España y Alemania es necesario prestar atención, por último, a la tasa de asalarización.

\subsection{Tasa de asalarización}

Es un ratio que se define como la proporción de trabajadores asalariados sobre el total de trabajadores, y que se utiliza para ponderar el coste laboral unitario por el número real de trabajadores asalariados existentes, excluyendo así a los trabajadores por cuenta propia.

Se trata de una variable estructural de las economías que no suele sufrir incrementos fuertes en el corto plazo, sino más bien movimientos de largo plazo alineados con la estructura del mercado de trabajo de cada economía en cada momento histórico. Estos movimientos también pueden ser provocados por modificaciones legislativas en materia laboral pero tardan un tiempo en hacer efecto.

En el caso de Alemania la tasa de asalarización ha permanecido relativamente estable a lo largo de todo nuestro periodo de estudio situándose en valores cercanos al $84-85 \%$. Durante la época de crisis sufrió descensos coyunturales de en torno a un punto porcentual, pero que se recuperaron rápidamente para situarse en el año 2013 en un 84,5\%. Los sectores alemanes con una mayor tasa de asalarización son: industria extractiva (98\%), suministros de electricidad, gas y agua (97\%) y manufacturas (95\%). España viene experimentando desde 1999 un progresivo aumento de su tasa de asalarización, que la hace pasar de un $81 \%$ a un $84,5 \%$, hasta frenarse en el año 2007. A partir de entonces la ratio se estabiliza y sufre un ligero aumento de medio punto porcentual para situarse en 2013 en el 85\%. Los sectores españoles con una mayor tasa de asalarización son: educación (97\%), salud (95\%) y manufacturas (91\%).Tras la observación de los gráficos y las tasas de variación podemos inferir que la tasa de asalarización en ambos países no ha incidido de manera sustancial en la evolución de la PS.

Como hemos señalado anteriormente, a partir del año 2010 se empiezan a dar las condiciones para la divergencia en las participaciones salariales de Alemania y España. La fuerte reducción del salario medio junto con el estancamiento de la productividad provocaron que la trayectoria seguida por la PS en España se alejase de la alemana.

Los mayores niveles de PS en Alemania se podrían deber a que los superiores niveles de productividad que ostenta la economía germana dejan un mayor margen para el incremento del salario medio y por tanto para un incremento de la PS. Ocurre siempre y cuando el crecimiento de los beneficios empresariales sea inferior al crecimiento de los salarios. En cambio la especialización de economías como la española en actividades de bajo valor añadido es posible que reporte unos niveles menores de PS, ya que los reducidos ratios de productividad hacen necesario un mayor ajuste en términos salariales, más aún 
Baratas, Luis. Distribución funcional de la renta: análisis de la divergencia en la participación salarial entre Alemania y España 1999 - 2013.

en un contexto de imposibilidad de devaluación externa. Esta idea sostendría nuestra opinión de que la introducción del euro ha servido para reforzar las asimetrías productivas y comerciales que, entre otras muchas consecuencias, conducen a una evolución divergente de la PS.

Asegurar esto no es equivalente a sostener que si no se llega a introducir el euro estas divergencias no hubieran tenido cabida, y por tanto la evolución de las participaciones salariales hubiese sido homogénea al alza. Las contradicciones más profundas referentes al conflicto capital-trabajo inherentes al sistema capitalista, junto a la feroz competencia a escala mundial cancelan esta hipótesis. Pero sí sostenemos que la no adopción del euro -o en otro caso, un diseño institucional alternativo de la eurozona y la Unión Europea- sí podía haber abierto un abanico de posibilidades diferentes al existente para actuar estratégicamente sobre los desequilibrios productivos y comerciales que separan a los países. Y por tanto haber provocado una evolución distinta de las participaciones salariales.

En cualquier caso, desde nuestro punto de vista, creemos que los motivos que explican las tendencias causantes de la divergencia en la PS (estancamiento de la productividad y fuerte reducción del salario medio), tienen una vinculación muy directa con la frágil y dependiente estructura productiva española y el debilitamiento de los sistemas de negociación colectiva. Aun así, insistimos en que contrastar que estas, y no otras, son las causas definitivas queda pendiente para un trabajo futuro.

\section{CONCLUSIONES}

El descenso de la PS durante las últimas décadas en las economías avanzadas es un hecho incontestable que se certifica con la mera observación de los datos. Dentro de las economías avanzadas hemos decidido centrar la atención de este trabajo en las economías europeas, concretamente la española y la alemana, por ser uno de los casos más significativos de descenso de la PS. Estos países que, a pesar de las diferencias existentes, han tenido históricamente una evolución similar de la PS han comenzado a experimentar una evolución divergente de la misma, por lo que el interés por identificar los factores explicativos que operan en uno y otro caso es doble. Además el hecho de que ambos países estén encuadrados en una misma unión monetaria como la europea suscita una mayor curiosidad intelectual si cabe.

La revisión de la literatura realizada al comienzo de este trabajo nos encamina hacia una previa concepción social del fenómeno de la distribución factorial de renta. Este proceso debe ser entendido como el resultado de un conflicto social definido por los equilibrios de poder entre las distintas clases sociales que participan en el proceso de producción, distribución y consumo. Solo así nos podremos aproximar adecuadamente a un análisis de sus factores explicativos y a una correcta interpretación de sus resultados empíricos. Al rechazar aquellos planteamientos teóricos que no asumen esta premisa -los planteamientos 
Baratas, Luis. Distribución funcional de la renta: análisis de la divergencia en la participación salarial entre Alemania y España 1999 - 2013.

neoclásicos- acudimos a diferentes corrientes de pensamiento donde hemos identificado multitud de factores explicativos de la DFR y en particular de la PS.

Todos los factores reconocidos canalizan su efecto sobre la PS a través de las variaciones que son capaces de provocar en los niveles de productividad y en el poder de negociación, entendido como el elemento que permite a los trabajadores beneficiarse en mayor o menor medida de dichos incrementos. De manera que, dado que consideramos que la productividad de un país está intrínsecamente unida la composición de su estructura productiva y que el establecimiento de los salarios está profundamente condicionado por el poder de negociación de los trabajadores; sostenemos la idea de que, dentro de una zona monetaria no óptima como la europea, la débil estructura productiva española, que no permite aumentos reales de productividad, junto con las restricciones a la negociación colectiva, que reducen los salarios relativos, son, entre otros factores, responsables de la divergencia que se produce en la PS entre España y Alemania. Aun así sería necesario un análisis más exhaustivo tanto de la estructura productiva como de los procesos de negociación colectiva en ambos países.

Los datos expuestos nos muestran como el descenso de la PS compartido por ambos países durante la etapa pre- crisis fue más intenso en Alemania que en España. Durante esta etapa el descenso de la PS en España se explica por un distanciamiento en los ritmos de crecimiento que experimentaron el salario medio y la productividad, fuertemente influenciados por el enérgico crecimiento de los precios que provocaba el modelo español. Incluso, el crecimiento de la tasa de asalarización moderó el descenso de la PS, que podía haber sido superior. Mientras tanto la economía alemana compaginó incrementos reales de productividad con políticas de moderación salarial, sin apenas incidencia de la tasa de asalarización, lo que derivó en una intensa reducción de la PS de mayor profundidad que la española.

A partir del año 2010 España no pudo continuar postergando el intenso declive de la PS al que estaba estructuralmente abocada, y a partir del año 2010 las políticas de ajuste salarial y el estancamiento de la productividad nominal condujeron a un acelerado declive de la PS que le alejó de la tendencia que comenzaría a experimentarse en Alemania. Gracias al comportamiento alcista de los salarios y de la productividad que permitía la estructura productiva alemana, junto con el leve incremento de la tasa de asalarización, la PS en Alemania comienza a crecer tenuemente y a divergir fuertemente de la tendencia española.

Finalmente los datos nos han revelado como la divergencia entre España y Alemania a partir de 2010 tiene sus raíces en una fuerte reducción de los salarios y un estancamiento de la productividad en España. En este sentido la incidencia de la débil estructura productiva española sobre la evolución de la productividad, el empleo y los salarios ha sido un elemento diferenciador de la tendencia seguida por Alemania, donde una mayor vigorosidad de la estructura productiva ha permitido unos niveles y unas variaciones superiores. A su vez el 
Baratas, Luis. Distribución funcional de la renta: análisis de la divergencia en la participación salarial entre Alemania y España 1999 - 2013.

debilitamiento de la negociación colectiva ha reducido el poder de negociación de los trabajadores, y con él la capacidad de negociar incrementos salariales. Nuestra consideración final es que ambas dimensiones señaladas tienen un papel fundamental en la evolución divergente de la PS como se ha demostrado con el análisis empírico.

Queremos advertir al lector por última vez que la intención originaria de este trabajo no era la de contrastar en sentido estricto las hipótesis explicativas que aquí se mencionan, sino la de analizar la evolución de las variables y apuntar varias posibles explicaciones a la divergencia en las participaciones salariales (débil estructura productiva y los cambios en la negociación colectiva en parte como consecuencia de la puesta en marcha del euro). Dejamos su contrastación como tal para un trabajo de investigación más amplio. 
Baratas, Luis. Distribución funcional de la renta: análisis de la divergencia en la participación salarial entre Alemania y España 1999 - 2013.

\section{BIBLIOGRAFÍA}

Álvarez, I., Luengo, F. \& Uxó, J. (2013). Fracturas y crisis en Europa, 1a ed. edn, Clave Intelectual; Eudeba, Madrid y Buenos Aires.

Arpaia, A., Pérez, E. \& Pichelmann, K. (2009). "Understanding labour income share dynamics in Europe", Economic Papers 379, Brussels.

Arrizabalo Montoro, X. (2014). Capitalismo y economía mundial: bases teóricas y análisis empírico para la comprensión de los problemas económicos del siglo XXI, Instituto Marxista de Economía, Madrid.

Azmat, G., Manning, A. \& Van Reenen, J. (2012). "Privatization and the decline of labour's share: international evidence from network industries", Economica, vol. 79, no. 315, pp. 470-492.

Bispinck, R. \& Schulten, T. 2012, "Collectively agreed wages in Germany", CAWIE paper. Leuven: KU Leuven/HIVA.

Blanchard, O., \& Giavazzi, F. (2003). Macroeconomic effects of regulation and deregulation in goods and labor markets (No. w8120). National Bureau of Economic Research.

Bogliciano, F. (2009). "Poorer workers. The determinants of wage formation in Europe", International Review of Applied Economics, vol. 23, no. 3, pp. 327343.

Boulding, K.E. (1950). A reconstruction of economics, John Wiley and Sons; Chapman and Hall, New York y London.

Ellis, L. \& Smith, K. (2007). The global upward trend in the profit share, Bank for International Settlement, BIS, Monetary and Economic Department.

European Commision, E. (2007). "The Labour Income Share in the European Union", Employment in Europe, vol. Chapter 5.

Felipe, J. \& Kumar, U. (2011). "Unit labor costs in the Eurozone: the competitiveness debate again", Levy Economics Institute of Bard College Working Paper, no. 651.

Fernández, M.J. (2012). "La distribución funcional de la renta en España y en la UEM en la última década", Cuadernos de Información económica, no. 231, pp. 33-38.

Garzón Espinosa, E. (2014). "Especialización productiva y comportamiento exportador de la economía alemana (1999-2007)". Trabajo final de Máster, Madrid: Universidad Complutense de Madrid (Departamento Economía Aplicada I).

Giammarioli, N., Messina, J., Steinberger, T. \& Strozzi, C. (2002). "European labor share dynamics: An institutional perspective".

International Labour Office, I. (2011). "The labour share of income: Determinants and potential contribution to exiting the financial crisis. Matthieu Charpe", World of Work Report, vol. 2011, no. 1, pp. 55-74.

International Monetary Fund, I. (2007). "The Globalization of Labour", World Economic Outlook. Washington, vol. Chapter 5.

Kaldor, N. (1955). "Alternative theories of distribution", The Review of Economic Studies, pp. 83-100.

Kalecki, M. (1954). Theory of Economic Dynamics: an easy on cyclical and longrun cahnges in capitalist Economy. Unwin Brothers Limited, pp: 1-44. 
Baratas, Luis. Distribución funcional de la renta: análisis de la divergencia en la participación salarial entre Alemania y España 1999 - 2013.

Kalecki, M. (1938). "The Determinants of Distribution of the National Income", Econometrica, vol. 6, no. 2, pp. 97-112.

Karanassou, M. \& Sala, H. (2013). "Inequality and employment sensitivities to the falling labour share", The Economic and Social Review, vol. 43, no. 3, Autumn, pp. 343-376.

Keynes, J.M. (2011). Teoría general de la ocupación, el interés y el dinero, Ciro, Barcelona.

Keynes, J.M. (1958). A treatise on money, Macmillan, London.

King, J.E. (2009). Historia de la economía poskeynesiana desde 1936, Ediciones AKAL.

Köhler, H. \& Calleja Jiménez, J.P. (2008). "Los sindicatos en España frente a los retos de la globalización y del cambio tecnológico".

Laborda Peralta, A. (2001). "La distribución funcional de la renta en España: una visión macroeconómica", Papeles de economía española, no. 88, pp. 4466.

Marchal, J. \& Lecaillon, J. (1958). La répartition du revenu national, M.-Th. Génin.

Marx, K. (2009). El Capital: crítica de la economía política. 8a. reimp. Edn, Siglo Veintiuno, Madrid.

Onaran, Ö. \& Galanis, G. (2013). "Is aggregate demand wage-led or profit-led? A global model", Wage-led Growth: An Equitable Strategy for Economic Recovery, Basingstoke, UK: Palgrave Macmillan, pp. 71-99.

Pérez Infante, J.I. (2012). "Reformas laborales, negociación colectiva y salarios", TES: EVALU,

Reed, H. \& Latorre, M. (2009). "The economic impacts of migration on the UK labour market", Institute for Public Policy Research, vol. 26.

Ricardo, D. (1994). Principios de economía política y tributación, 1ae ed., 5ª reimp. Edn, Cambridge University Press, México.

Rodríguez, E. \& López, I. (2011). "Del auge al colapso. El modelo financieroinmobiliario de la economía española (1995-2010)", Revista de economía crítica, no. 12, pp. 39-63.

Ruza Tarrio, J. (1974). Teorías macroeconómicas de la distribución funcional de la renta, Revista de Economía Política.

Stockhammer, E. (2013). "Why have wage shares fallen? A panel analysis of the determinants of functional income distribution". International Labour Organisation (ILO) Conditions of Work and Employment Series, no 35".

Verduzco, M.A. (1998). Crecimiento económico y distribución del ingreso: balance teórico y evidencia empírica, UNAM.

Weintraub, S. (1958). An approach to the theory of income distribution, Chilton Company, Philadelphia.

Young, A.T. (2004). "Labors share fluctuations, biased technical change, and the business cycle", Review of economic dynamics, vol. 7, no. 4, pp. 916-931. 
Baratas, Luis. Distribución funcional de la renta: análisis de la divergencia en la participación salarial entre Alemania y España 1999 - 2013.

\section{Anexo 1. Cuadro resumen: Tasas de variación interanual de las variables según subperiodos, 1999 - 2013}

\begin{tabular}{|c|c|c|c|c|}
\hline \multirow[b]{2}{*}{ Etapa pre-crisis } & \multicolumn{4}{|c|}{$1999-2007$} \\
\hline & $\begin{array}{c}1999-2003 \\
\text { ESP }\end{array}$ & $\begin{array}{c}1999-2003 \\
\text { ALE }\end{array}$ & $\begin{array}{c}2004-2007 \\
\text { ESP }\end{array}$ & $\begin{array}{c}2004-2007 \\
\text { ALE }\end{array}$ \\
\hline Compensación empleados & $7,18 \%$ & $1,61 \%$ & $7,38 \%$ & $0,93 \%$ \\
\hline Empleo asalariado & $3,50 \%$ & $-0,80 \%$ & $3,14 \%$ & $0,27 \%$ \\
\hline Salario medio & $3,55 \%$ & $2,43 \%$ & $4,11 \%$ & $0,65 \%$ \\
\hline Producción & $7,80 \%$ & $1,80 \%$ & $7,69 \%$ & $3,13 \%$ \\
\hline Empleo total & $2,99 \%$ & $-0,81 \%$ & $2,76 \%$ & $0,36 \%$ \\
\hline Productividad trabajo & $4,67 \%$ & $2,63 \%$ & $4,80 \%$ & $2,76 \%$ \\
\hline CLUr & $-1,07 \%$ & $-0,19 \%$ & $-0,66 \%$ & $-2,05 \%$ \\
\hline Tasa asalarización & $0,50 \%$ & $0,01 \%$ & $0,38 \%$ & $-0,09 \%$ \\
\hline \multirow[t]{3}{*}{ PART. SAL } & $-0,58 \%$ & $-0,18 \%$ & $-0,28 \%$ & $-2,13 \%$ \\
\hline & \multicolumn{4}{|c|}{$2008-2013$} \\
\hline & $\begin{array}{c}2008-2010 \\
\text { ESP }\end{array}$ & $\begin{array}{c}2008-2010 \\
\text { ALE }\end{array}$ & $\begin{array}{c}2010-2013 \\
\text { ESP }\end{array}$ & $\begin{array}{c}2010-2013 \\
\text { ALE }\end{array}$ \\
\hline Compensación empleados & $0,78 \%$ & $2,25 \%$ & $-3,26 \%$ & $3,70 \%$ \\
\hline Empleo asalariado & $-2,71 \%$ & $0,28 \%$ & $-0,75 \%$ & $0,93 \%$ \\
\hline Salario medio & $3,57 \%$ & $2,00 \%$ & $-2,53 \%$ & $2,74 \%$ \\
\hline Producción & $-0,20 \%$ & $0,98 \%$ & $-0,72 \%$ & $3,15 \%$ \\
\hline Empleo total & $-2,73 \%$ & $0,21 \%$ & $-0,94 \%$ & $0,64 \%$ \\
\hline Productividad trabajo & $2,60 \%$ & $0,73 \%$ & $0,22 \%$ & $2,48 \%$ \\
\hline CLUr & $0,94 \%$ & $1,32 \%$ & $-2,75 \%$ & $0,26 \%$ \\
\hline Tasa asalarización & $0,02 \%$ & $0,06 \%$ & $0,19 \%$ & $0,29 \%$ \\
\hline PART. SAL & $0,96 \%$ & $1,36 \%$ & $-2,57 \%$ & $0,55 \%$ \\
\hline
\end{tabular}

\section{Anexo 2. Resumen gráfico de la PS y sus componentes, 1999 - 2013}

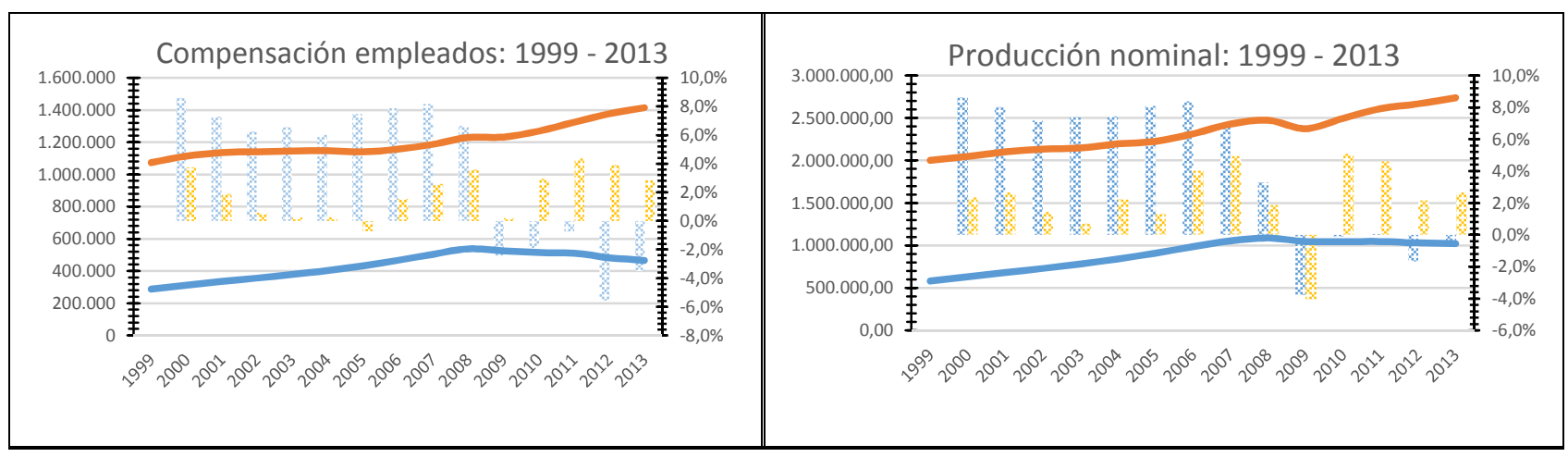

Papeles de Europa

Vol. 28, Núm. 2 (2015): 54-89 http://dx.doi.org/10.5209/rev_PADE.2015.v28.n2.51903 
Baratas, Luis. Distribución funcional de la renta: análisis de la divergencia en la participación salarial entre Alemania y España 1999 - 2013.

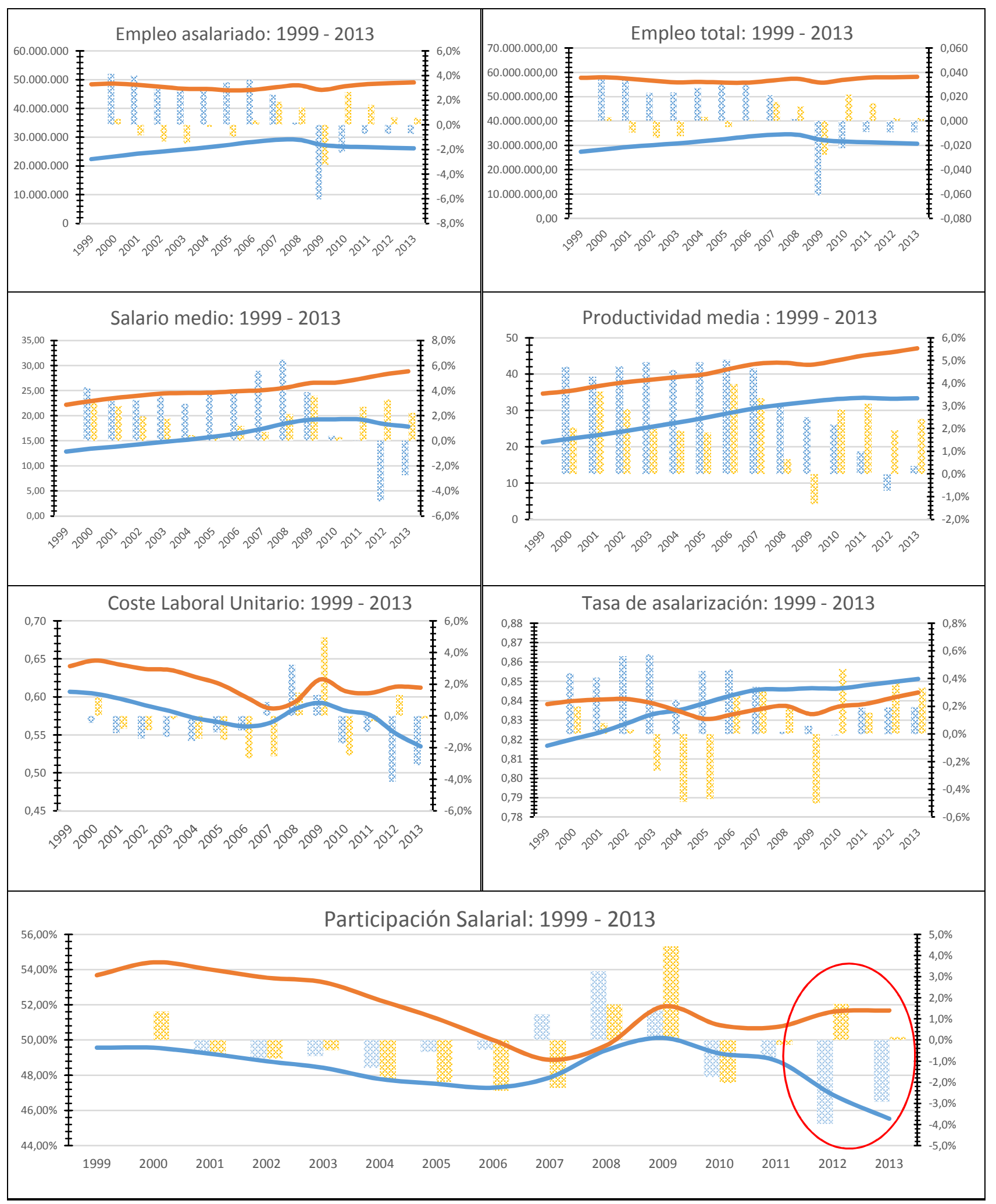

Fuente: elaboración propia a partir de Eurostat. 